\title{
On localized signature and higher rho invariant of fibered manifolds
}

\author{
Liu Hongzhi ${ }^{1}$ and Wang Jinmin ${ }^{2}$ \\ ${ }^{1}$ School of Mathematics, Shanghai University of Finance and \\ Economics, \\ Shanghai 200433, P. R. China. \\ e-mail: liu.hongzhi@mail.shufe.edu.cn \\ ${ }^{2}$ Shanghai Center for Mathematical Sciences, Fudan University, \\ Shanghai 200433, P. R. China. \\ e-mail: wangjm15@fudan.edu.cn
}

\begin{abstract}
Higher index of signature operator is a far reaching generalization of signature of a closed oriented manifold. When two closed oriented manifolds are homotopy equivalent, one can define a secondary invariant of the relative signature operator called higher rho invariant. The higher rho invariant detects the topological nonrigidity of a manifold. In this paper, we prove product formulas for higher index and higher rho invariant of signature operator on fibered manifolds. Our result implies the classical product formula for numerical signature of fiber manifolds obtained by Chern, Hirzebruch, and Serre in [1]. We also give a new proof of the product formula for higher rho invariant of signature operator on product manifolds, which is parallel to the product formula for higher rho invariant of Dirac operator on product manifolds obtained by Xie and $\mathrm{Yu}$ in [14] and Zeidler in [17].
\end{abstract}

\section{Introduction}

The signature of a $4 k$-dimensional manifold is defined to be the signature of the cup product as a non-degenerate symmetric bilinear form on the vector space 
of $2 k$-cohomology classes. In [1], Chern, Hirzebruch and Serre established a product formula of signature for fibered manifold. More precisely, let $F \rightarrow$ $E \rightarrow B$ be a fibered manifold with base manifold $B$ and fiber manifold $F$, if $\pi_{1}(B)$ acts trivially on $H_{d R}^{*}(F)$, the de Rham cohomology of $F$. We have the following product formula

$$
\operatorname{sgn}(B) \times \operatorname{sgn}(F)=\operatorname{sgn}(E) .
$$

The signature of a manifold is also equal to the Fredholm index of the signature operator. When taking into account of the fundamental group of the manifold, one can introduce higher invariants of the signature operator, which lie in the $K$-theory of certain geometric $C^{*}$-algebras. Let $M$ be an $m$-dimensional manifold with fundamental group $\pi_{1}(M)=G$ and universal covering $\widetilde{M}$. Let $D_{M}^{s g n}$ be the signature operator on $M$. The higher index of $D_{M}^{s g n}, \operatorname{Ind}\left(D_{M}^{s g n}\right)$ is a generalization of the Fredholm index, and is defined to be an element in $K_{m}\left(C^{*}(\widetilde{M})^{G}\right)$, where $C^{*}(\widetilde{M})^{G}$ is the equivariant Roe algebra of $\widetilde{M}$ and is Morita equivalent to the reduced group $C^{*}$-algebra $C_{r}^{*}(G)$. The higher index of signature operator is invariant under homotopy equivalence and oriented cobordism, and plays a fundamental role in the study of classification of manifolds. On the other hand, $D_{M}^{s g n}$ defines a $K$-homology class $\left[D_{M}^{s g n}\right]$ in $K_{m}\left(C_{L}^{*}(\widetilde{M})^{G}\right)$, the $K$-theory of the equivariant localization algebra. See Section 2 and 3 for the explicit definitions of equivariant geometric $C^{*}$-algebras, higher index and $K$-homology class of signature operator.

Furthermore, if $f: M^{\prime} \rightarrow M$ is an orientation-preserving homotopy equivalence of closed manifolds, then there exists a concrete homotopy path that realizes the equality

$$
\operatorname{Ind}\left(D_{M^{\prime}}^{s g n}\right)=\operatorname{Ind}\left(D_{M}^{s g n}\right) \in K_{m}\left(C^{*}(\widetilde{M})^{G}\right),
$$

where $m$ is the dimension of $M$ and $M^{\prime}$. This homotopy path allows one to define a secondary invariant of signature operator associated to the homotopy equivalence $f$, called higher rho invariant, in the $K$-theory of the equivariant obstruction algebra $C_{L, 0}^{*}(\widetilde{M})^{G}$. The higher rho invariant of signature operator associated to homotopy equivalence plays a central role in estimating the topological nonrigidity of a manifold (cf: $[4,7,8,13,18]$ ).

Inspired by Chern, Hirzebruch and Serre's product formula, we prove a product formula for higher index and higher rho invariant of signature operator on fibered manifold. More precisely, consider a closed fibered manifold $F \rightarrow$ $E \rightarrow B$ with base space $B$ and fiber $F$. Denote the fundamental group of $E$ by $G$, and the fundamental group of $B$ by $H$. Let $\widetilde{E}$ and $\widetilde{B}$ be the universal covering spaces of $E$ and $B$ respectively. Set $n=\operatorname{dim} F$ and $m=\operatorname{dim} B$. 
We first define equivariant family localization algebra $C_{L}^{*}(\widetilde{E} ; \widetilde{B})^{G}$, and family obstruction algebra $C_{L, 0}^{*}(\widetilde{E} ; \widetilde{B})^{G}$. We show that there are naturally defined product maps:

$$
\begin{aligned}
\phi: K_{m}\left(C_{L}^{*}(\widetilde{B})^{H}\right) \otimes K_{n}\left(C_{L}^{*}(\widetilde{E}, \widetilde{B})^{G}\right) & \rightarrow K_{m+n}\left(C_{L}^{*}(\widetilde{E})^{G}\right), \\
\phi_{0}: K_{m}\left(C_{L}^{*}(\widetilde{B})^{H}\right) \otimes K_{n}\left(C_{L, 0}^{*}(\widetilde{E}, \widetilde{B})^{G}\right) & \rightarrow K_{m+n}\left(C_{L, 0}^{*}(\widetilde{E})^{G}\right) .
\end{aligned}
$$

Taking advantage of the fiberwise signature operator, we introduce the family $K$-homology class of family signature operator along $F$, denoted by $\left[D_{E, B}^{s g n}\right]$, in the $K$-theory of the equivariant family localization algebra, and the family higher rho invariant $\rho(f ; B)$, associated to a fiberwise homotopy equivalence $f: E^{\prime} \rightarrow E$, in the $K$-theory of the equivariant family obstruction algebra. The following theorem is a product formula for $K$-homology class of signature operator on fibered manifold, which implies the product formula for higher index of signature operator.

Theorem 1.1. Let $F \rightarrow E \rightarrow B$ be fibered manifold with base space $B$ and fiber $F$. Denote the fundamental group of $E$ by $G$, and the fundamental group of $B$ by $H$. Let $\widetilde{E}$ and $\widetilde{B}$ be the universal covering spaces of $E$ and $B$ respectively. Write $\operatorname{dim} F=n$ and $\operatorname{dim} B=m$. Let $\left[D_{E, B}^{s g n}\right]$ be the family $K$-homology class of the family signature operator in $K_{n}\left(C_{L}^{*}(\widetilde{E}, \widetilde{B})^{G}\right)$. We have the following product formula for family $K$-homology class of family signature operator

$$
k_{m n} \cdot \phi\left(\left[D_{B}^{s g n}\right] \otimes\left[D_{E, B}^{s g n}\right]\right)=\left[D_{E}^{s g n}\right],
$$

where $k_{m n}=1$ when $m n$ is even and $k_{m n}=2$ otherwise, and $\phi$ is the product map

$$
\phi: K_{m}\left(C_{L}^{*}(\widetilde{B})^{H}\right) \otimes K_{n}\left(C_{L}^{*}(\widetilde{E}, \widetilde{B})^{G}\right) \rightarrow K_{m+n}\left(C_{L}^{*}(\widetilde{E})^{G}\right) .
$$

We also obtain the following product formula for higher rho invariant of signature operator on fibered manifold.

Theorem 1.2. Let $F \rightarrow E \rightarrow B$ and $F^{\prime} \rightarrow E^{\prime} \rightarrow B$ be two fibered manifolds with base space $B$ and fiber $F$ and $F^{\prime}$ respectively. Let $f: E^{\prime} \rightarrow E$ be a fiberwise homotopy equivalence. Denote the fundamental group of $E$ and $E^{\prime}$ by $G$, and the fundamental group of $B$ by $H$. Let $\widetilde{E}, \widetilde{E^{\prime}}$ and $\widetilde{B}$ be the universal covering spaces of $E, E^{\prime}$ and $B$ respectively. Write $\operatorname{dim} F=n$ and $\operatorname{dim} B=m$. Let $\rho(f ; B)$ be the family higher rho invariant associated to fiberwise homotopy equivalence $f$ defined in $K_{n}\left(C_{L, 0}^{*}(\widetilde{E}, \widetilde{B})^{G}\right)$. We have the following product formula for higher rho invariant associated to fiberwise homotopy equivalence

$$
k_{m n} \cdot \phi_{0}\left(\left[D_{B}^{s g n}\right] \otimes \rho(f ; B)\right)=\rho(f),
$$


where $k_{m n}=1$ when $m n$ is even and $k_{m n}=2$ otherwise, and $\phi_{0}$ is the product map

$$
\phi_{0}: K_{m}\left(C_{L}^{*}(\widetilde{B})^{H}\right) \otimes K_{n}\left(C_{L, 0}^{*}(\widetilde{E}, \widetilde{B})^{G}\right) \rightarrow K_{m+n}\left(C_{L, 0}^{*}(\widetilde{E})^{G}\right) .
$$

As an application of Theorem 1.1, we give an alternative proof of the product formula of Chern, Hirzebruch and Serre (cf: [1]). Also, the product formula of higher rho invariant stated in Theorem 1.2 can be applied to study the behavior of higher rho map in [13] under fibration, and thus can be applied to study the topological nonrigidty of fibered manifold.

We mention that the product formula for higher index of signature operator has been obtained by Wahl in [11]. In this paper, we give a new proof of Wahl's product formula. On the other hand, product formula for higher rho invariant for positive scalar curvature metric on product manifolds has been proved by Siegel in his thesis [9], by Xie and Yu in [14], and by Zeidler in [17]. Their results and Theorem 6.8, 6.9 in [13] inspire us to study the product formula for higher rho invariant for signature operators.

The paper is organized as follows. In Section 2, we briefly recall some definitions of geometric $C^{*}$-algebras that we may use throughout the paper. In Section 3, we revisit the construction of several higher invariants associated to the signature operator. Next in Section 4, we prove the product formulas for higher index and higher rho invariant of signature operator on product manifolds. In Section 5, we generalize the product formulas to fibered manifold and prove Theorem 1.1 and 1.2. We shall define an auxiliary $C^{*}$-algebra consisting of operators that can be localized along the base manifold, and use the Mayer-Vietoris arguments.

\section{Preliminary}

The aim of this section is to briefly recall some basic definitions of geometric $C^{*}$-algebras used throughout the paper. For more details, we refer the readers to $[13]$.

Let $X$ be a proper metric space and $G$ be a finitely represented discrete group. Suppose that $G$ acts on $X$ properly by isometries. For simplicity, we assume that the $G$-action is free. Let $C_{0}(X)$ be the $C^{*}$-algebra consisting of all complex-valued continuous functions on $X$ that vanish at infinity. An $X$-module is a separable Hilbert space $H_{X}$ equipped with a $*$-representation of $C_{0}(X)$. It is called nondegenerate if the $*$-representation is nondegenerate, and standard if no nonzero function in $C_{0}(X)$ acts as a compact operator. Additionally we assume that $H_{X}$ is equipped with a unitary representation of 
$G$ which is compatible with $C_{0}(X)$-representation, that is,

$$
\forall f \in C_{0}(X), g \in G, \pi(g) \phi(f)=\phi(g . f) \pi(g)
$$

where $\phi($ resp. $\pi)$ is the $C_{0}(X)($ resp. $G)$-representation on $H_{X}$ and $g . f(x)=$ $f\left(g^{-1} x\right)$.

Now let us recall the definitions of propagation of operator and locally compact operator.

Definition 2.1. Under the above assumptions, let $T$ be a bounded linear operator acting on $H_{X}$.

1. The propagation of $T$ is defined by

$$
\operatorname{prop}(T)=\sup \{d(x, y) \mid(x, y) \in \operatorname{Supp}(T)\},
$$

where $\operatorname{Supp}(T)$ is the complement (in $X \times X)$ of the set of points $(x, y) \in$ $X \times X$ such that there exists $f_{1}, f_{2} \in C_{0}(X)$ such that $f_{1} T f_{2}=0$ and $f_{1}(x) f_{2}(y) \neq 0$;

2. $T$ is said to be locally compact if both $f T$ and $T f$ are compact for all $f \in C_{0}(X)$.

In the following, we recall the definitions of the equivariant Roe algebra, localization algebra, and the obstruction algebra.

Definition 2.2. Let $H_{X}$ be a standard nondegenerate $X$-module and $B\left(H_{X}\right)$ the set of all bounded linear operators on $H_{X}$.

1. The $G$-equivariant Roe algebra of $X$, denoted by $C^{*}(X)^{G}$, is the $C^{*}$ algebra generated by all $G$-equivariant locally compact operators with finite propagation in $B\left(H_{X}\right)$.

2. The $G$-equivariant localization algebra $C_{L}^{*}(X)^{G}$ is the $C^{*}$-algebra generated by all uniformly norm-bounded and uniformly norm-continuous functions $f:[1, \infty) \rightarrow C^{*}(X)^{G}$ such that

$$
\operatorname{prop}(f(t))<\infty \text { and } \operatorname{prop}(f(t)) \rightarrow 0 \text { as } t \rightarrow \infty \text {. }
$$

3. The $G$-equivariant obstruction algebra $C_{L, 0}^{*}(X)^{G}$ is defined to be the kernel of the following evaluation map

$$
\begin{aligned}
\mathrm{ev}: C_{L}^{*}(X)^{G} & \rightarrow C^{*}(X)^{G} \\
f & \mapsto f(1)
\end{aligned}
$$

In particular, $C_{L, 0}^{*}(X)^{G}$ is an ideal of $C_{L}^{*}(X)^{G}$. 
Remark 2.3. Up to isomorphism, $C^{*}(X)^{G}$ does not depend on the choice of the standard nondegenerate $X$-module. The same holds for $C_{L}^{*}(X)^{G}$ and $C_{L, 0}^{*}(X)^{G}$.

When $X$ is a Galois $G$-covering of a closed Riemannian manifold, $L^{2}(X)$ is a standard nondegenerate $X$-module. In this case, there is an equivalent definition of equivariant Roe algebra.

Definition 2.4. Let $X$ be a Galois $G$-covering of a closed Riemannian manifold. Set $\mathbb{C}[X]^{G}$ as a *-algebra consisting of integral operators given by

$$
\forall f \in L^{2}(X), f \mapsto \int_{X} k(x, y) f(y) d y
$$

where $k: X \times X \rightarrow \mathbb{C}$ is uniformly continuous, bounded on $X \times X$, and has finite propagation, i.e.

$$
\exists \delta>0 \text {, s.t. } k(x, y)=0 \text {, if } d(x, y)>\delta,
$$

and is $G$-equivariant, i.e. $k(g x, g y)=k(x, y)$ for any $g \in G$. The $G$-equivariant equivariant Roe algebra is the operator norm completion of $\mathbb{C}[X]^{G}$.

Remark 2.5. If we remove the cocompactness of the $G$-action, Definition 2.4 actually gives us the uniform equivariant Roe algebra, which is different from the equivariant Roe algebra.

Suppose that $T \in \mathbb{C}[X]^{G}$ has corresponding Schwartz kernel $k(x, y)$. The support of $T$ defined in Definition 2.2 is simply given by

$$
\operatorname{Supp}(T)=\overline{\{(x, y) \in X \times X: k(x, y) \neq 0\}} .
$$

Similarly we define the $G$-equivariant localization algebra $C_{L}^{*}(X)^{G}$ to be the completion of all paths on $t \in[0,+\infty)$ with value in $\mathbb{C}[X]^{G}$, which are uniformly continuous and uniformly bounded with respect to the operator norm, and have propagation going to zero as $t$ goes to infinity.

Definition 2.4 can be easily generalized to the case where $L^{2}(X)$ is replaced by $L^{2}$-section of an Hermitian vector bundle over $X$ on which $G$ acts by isometries. The above definitions coincide with Definition 2.2, as those $C^{*}$-algebras are independent of the choice of the standard nondegenerate $X$-module.

Now we consider a product of two manifolds. Let $M, N$ be two closed manifolds and $\widetilde{M}, \widetilde{N}$ be their Galois $G, H$-covering spaces respectively. For any pair of integral operators in $\mathbb{C}[\widetilde{M}]^{G}$ and $\mathbb{C}[\tilde{N}]^{H}$, their tensor product is well-defined as an operator in $\mathbb{C}[\widetilde{M} \times \tilde{N}]^{G \times H}$. This induces the following 
product maps

$$
\begin{gathered}
\psi: K_{m}\left(C^{*}(\widetilde{M})^{G}\right) \otimes K_{n}\left(C^{*}(\tilde{N})^{H}\right) \rightarrow K_{m+n}\left(C_{L}^{*}(\widetilde{M} \times \tilde{N})^{G \times H}\right), \\
\psi_{L}: K_{m}\left(C_{L}^{*}(\widetilde{M})^{G}\right) \otimes K_{n}\left(C_{L}^{*}(\widetilde{N})^{H}\right) \rightarrow K_{m+n}\left(C_{L}^{*}(\widetilde{M} \times \tilde{N})^{G \times H}\right), \\
\psi_{L, 0}: K_{m}\left(C_{L}^{*}(\widetilde{M})^{G}\right) \otimes K_{n}\left(C_{L, 0}^{*}(\tilde{N})^{H}\right) \rightarrow K_{m+n}\left(C_{L, 0}^{*}(\widetilde{M} \times \widetilde{N})^{G \times H}\right) .
\end{gathered}
$$

\section{$3 \quad$ Higher invariant associated to signature operator}

In this section, we recall a formula of the higher index of signature operator, which was obtained by Higson and Roe in [2] and [3]. After that we will give the construction of $K$-homology class of the signature operator, which was originally introduced by Weinberger, Xie and Yu in [13]. At last, we give the definition of the higher rho invariant of controlled homotopy equivalence of manifolds.

In this section, all manifolds mentioned are not assumed to be compact or connected unless otherwise noted.

\subsection{Higher index of signature operator}

Let $M$ be a Riemannian manifold of dimension $m$. Let $\widetilde{M}$ be a Galois $G$ covering space of $M$, where $G$ is a finitely represented discrete group. Denote by $\Lambda^{p}(\widetilde{M})$ the $L^{2}$-completion of compactly support smooth differential $p$-forms on $\widetilde{M}$. Let $d_{\widetilde{M}}$ be the usual differential operators, which is an unbounded operator from $\Lambda^{p}(\widetilde{M})$ to $\Lambda^{p+1}(\widetilde{M})$. We will write $\Lambda^{\text {even }}(\widetilde{M})=\oplus_{k} \Lambda^{2 k}(\widetilde{M})$, $\Lambda^{\text {odd }}(\widetilde{M})=\oplus_{k} \Lambda^{2 k+1}(\widetilde{M})$, and $\Lambda(\widetilde{M})=\Lambda^{\text {even }}(\widetilde{M}) \oplus \Lambda^{\text {odd }}(\widetilde{M})$. as

Set $D_{\widetilde{M}}=d_{\widetilde{M}}+d_{\widetilde{M}}^{*}$. Let $*$ be the Hodge $*$-operator of $\Lambda^{*}(\widetilde{M})$. Define $S_{\widetilde{M}}$

$$
\begin{aligned}
S_{\widetilde{M}}: \Lambda^{p}(\widetilde{M}) & \rightarrow \Lambda^{n-p}(\widetilde{M}), \\
\omega & \rightarrow i^{p(p-1)+\left[\frac{n}{2}\right]} * \omega .
\end{aligned}
$$

We now recall the representative of higher index of signature operator according to the parity of $m$.

Odd case. When $m$ is odd, the signature operator $D_{M}^{s g n}$ is given by

$$
i D_{\widetilde{M}} S_{\widetilde{M}}: \Lambda^{\text {even }}(\widetilde{M}) \rightarrow \Lambda^{\text {even }}(\widetilde{M}) .
$$

It is shown in [2] and [3] that 
1. $D_{\widetilde{M}} \pm S_{\widetilde{M}}$ are both invertible;

2. the invertible operator

$$
\left(D_{\widetilde{M}}+S_{\widetilde{M}}\right)\left(D_{\widetilde{M}}-S_{\widetilde{M}}\right)^{-1}: \Lambda^{e v e n}(\widetilde{M}) \rightarrow \Lambda^{e v e n}(\widetilde{M})
$$

belongs to $\left(C^{*}(\widetilde{M})^{G}\right)^{+}$, thus defines a class in $K_{1}\left(C^{*}(\widetilde{M})^{G}\right)$ denoted by $\operatorname{Ind}\left(D_{M}^{s g n}\right)$;

3. the higher index of the signature operator is equal to $\operatorname{Ind}\left(D_{M}^{s g n}\right)$.

Even case. The even case is parallel. When $m$ is even, the signature operator $D_{M}^{s g n}$ is $D_{\widetilde{M}}$ together with the grading operator $S_{\widetilde{M}}$.

It is shown in [2] and [3] that

1. $D_{\widetilde{M}} \pm S_{\widetilde{M}}$ are both invertible;

2. Let $P_{+}\left(D_{\widetilde{M}} \pm S_{\widetilde{M}}\right)$ be the positive projections of invertible operators $D_{\widetilde{M}} \pm S_{\widetilde{M}}$ respectively. Then $P_{+}\left(D_{\widetilde{M}} \pm S_{\widetilde{M}}\right)$ can be approximated by operators with finite propagation, and

$$
P_{+}\left(D_{\widetilde{M}}+S_{\widetilde{M}}\right)-P_{+}\left(D_{\widetilde{M}}-S_{\widetilde{M}}\right) \in C^{*}(\widetilde{M})^{G},
$$

thus the formal difference

$$
\left[P_{+}\left(D_{\widetilde{M}}+S_{\widetilde{M}}\right)\right]-\left[P_{+}\left(D_{\widetilde{M}}-S_{\widetilde{M}}\right)\right]
$$

determines a $K$-theory class in $K_{0}\left(C^{*}(\widetilde{M})^{G}\right)$ denoted by $\operatorname{Ind}\left(D_{M}^{s g n}\right)$;

3. the higher index of the signature operator is equal to $\operatorname{Ind}\left(D_{M}^{s g n}\right)$.

\section{2 $K$-homology class of signature operator}

In this subsection we recall the definition of $K$-homology class of signature operator according to the parity of $\operatorname{dim} M$.

Odd case. Suppose that $M$ is odd dimensional. For any $n \in \mathbb{N}^{+}$, let $M_{n}$ be the manifold $M$ equipped with metric $g_{n}$, which is $n$-times of the original metric $g$. Denote by $\coprod_{n} M_{n}$ the disjoint union of $M_{n}$. The higher index of signature operator $D_{\amalg M_{n}}^{s g n}$ is represented by the invertible operator

$$
T=\left.\left(D_{\amalg_{n} \widetilde{M}_{n}}+S_{\amalg_{n} \widetilde{M}_{n}}\right)\left(D_{\amalg_{n} \widetilde{M}_{n}}-S_{\amalg_{n} \widetilde{M}_{n}}\right)^{-1}\right|_{\Lambda^{\text {even }}\left(\amalg_{n} \widetilde{M}_{n}\right)} \cdot
$$

Denote by $T_{n}$ the restriction of $T$ to $M_{n}$. As shown in the previous subsection, $T$ can be approximated by operators with finite propagation on $\coprod_{n} M_{n}$. 
Therefore with respect to the original metric $g,\left\{T_{n}\right\}$ can be approximated by a sequence of operators uniformly whose propagation goes to zero as $n$ goes to infinity.

To make the above sequence a continuous path on $[1,+\infty)$, we further consider $\coprod_{n} M_{n+r}$ for $r \in[0,1]$ rather than $\coprod_{n} M_{n}$, where the metric on $M_{n+r}$ is $(n+r)$-times of the original metric. This actually gives an invertible element in $C_{L}^{*}(\widetilde{M})^{G}$.

Definition 3.1. We call the $K$-theory element in $K_{1}\left(C_{L}^{*}(\widetilde{M})^{G}\right)$ represented by the invertible element defined above the $K$-homology class of signature operator, which will be denoted by $\left[D_{M}^{s g n}\right]$.

Even case. We sketch the construction of $K$-homology class of signature operator for the case of $m$ being even but leave out the details. The higher index of signature operator $D_{\amalg M_{n}}^{s g n}$ is determined by the difference of projections

$$
P_{+}\left(D_{\amalg \widetilde{M}_{n}}+S_{\amalg \widetilde{M}_{n}}\right)-P_{+}\left(D_{\amalg \widetilde{M}_{n}}-S_{\amalg \widetilde{M}_{n}}\right) .
$$

Again, we consider the higher index of signature operator on $\coprod_{n} M_{n+r}$ for $r \in[0,1]$. This gives us a continuous path from $[1,+\infty)$ to $C^{*}(\widetilde{M})^{G}$, which in turn defines a path of difference of projections lies in $C_{L}^{*}(\widetilde{M})^{G}$, denoted by $P_{M,+}(t)-P_{M,-}(t)$, with respect to the original metric $g$.

Definition 3.2. We call the $K$-theory element determined by the formal difference $\left[P_{M,+}(t)\right]-\left[P_{M,-}(t)\right]$ the $K$-homology class of signature operator $D_{M}^{s g n}$, which will be denoted by $\left[D_{M}^{s g n}\right] \in K_{0}\left(C_{L}^{*}(\widetilde{M})^{G}\right)$.

\subsection{Controlled homotopy equivalence and higher rho invariant}

In this subsection, we recall the construction of higher rho invariant of signature operator associated to homotopy equivalence.

Higher rho invariant associated to smooth homotopy equivalence was first introduced by Higson and Roe in [2-4]. Later in [8], Piazza and Schick gave an index theoretic definition of higher rho invariant of signature operator. In [18], Zenobi extended Higson and Roe, Piazza and Schick's work to define higher rho invariant associated to topological homotopy equivalence.

In [13], Weinberger, Xie and Yu constructed higher rho invariant of signature operator associated to homotopy equivalence by piecewise-linear approach. In this paper, we adapt their construction to give a differential geometric approach to the definition of higher rho invariant. It is not hard to see that our construction here is equivalent to the one given in [15]. 
Definition 3.3. Let $M^{\prime}$ and $M$ be two Riemannian manifolds. Let $f: M^{\prime} \rightarrow$ $M$ be a smooth homotopy equivalence with smooth homotopy inverse $g: M \rightarrow$ $M^{\prime}$. Denote by $h_{t}^{\prime}, t \in[0,1]$ (resp. $h_{t}, t \in[0,1]$ ) the smooth homotopy between $f g$ and $\operatorname{id}_{M^{\prime}}$ (resp. $g f$ and $\mathrm{id}_{M}$ ). We say that $f$ is a controlled homotopy equivalence if there exists a positive constant $C$ such that

1. the diameter of $\left\{h_{t}^{\prime}(a) \mid 0 \leqslant t \leqslant 1\right\}$ is bounded by $C$ uniformly for all $a \in M^{\prime}$,

2. the diameter of $\left\{h_{t}(b) \mid 0 \leqslant t \leqslant 1\right\}$ is bounded by $C$ uniformly for all $b \in M$.

Remark 3.4. If $M^{\prime}$ and $M$ are closed manifold, then any homotopy equivalence $f: M^{\prime} \rightarrow M$ is automatically controlled. Furthermore, the lift of $f$ to their Galois covering is also controlled.

Let $f: M^{\prime} \rightarrow M$ be a controlled homotopy equivalence. Suppose that $\widetilde{M^{\prime}}, \widetilde{M}$ are Galois $G$-covering spaces of $M, M^{\prime}$ respectively. Write $D=D_{\widetilde{M^{\prime}}} \oplus$ $D_{\widetilde{M}}$, and $S=\left(\begin{array}{ll}S_{\widetilde{M^{\prime}}} & \\ & -S_{\widetilde{M}}\end{array}\right)$ acting on $\Lambda^{*}\left(\widetilde{M}^{\prime}\right) \otimes \Lambda^{*}(\widetilde{M})$. The controlled homotopy equivalence $f: M^{\prime} \rightarrow M$ induces a map from $\Lambda^{p}(\widetilde{M})$ to $\Lambda^{p}\left(\widetilde{M^{\prime}}\right)$, which we will still denote by $f$. In general, the induced map is not a bounded operator. However, we may apply the Hilsum-Skandalis submersion (cf: [6, Page74], [12, Page 157], and [15, Page 34]) to construct a bounded operator $\mathcal{T}_{f}$ out of $f$. Without loss of generality, we might as well assume that $f$ is a bounded operator.

Now let us recall the definition of higher rho invariant associated to controlled homotopy equivalence $f$ according to the parity of $\operatorname{dim} M$. We mention here that the construction is due to Higson and Roe (cf: [2], [4]), and Weinberger, Xie and Yu (cf: [13]).

Odd case We first assume that both $M^{\prime}$ and $M$ are odd dimensional. Via conjugating by $f$ on the first summand $\Lambda^{*}\left(\widetilde{M}^{\prime}\right)$, we may identify $D$ and $S$ with their corresponding operators acting on $\Lambda^{*}(\widetilde{M}) \oplus \Lambda^{*}(\widetilde{M})$. Under this identification, the invertible element defined by

$$
\left.(D+S)(D-S)^{-1}\right|_{\Lambda^{\text {even }}\left(\widetilde{M^{\prime}}\right) \oplus \Lambda^{\text {even }}(\widetilde{M})} \in M_{2}\left(C_{L}^{*}(\widetilde{M})^{G}\right)^{+}
$$

represents the $K$-theory element $f_{*} \operatorname{Ind}\left(D_{M^{\prime}}^{s g n}\right)-\operatorname{Ind}\left(D_{M}^{s g n}\right) \in K_{1}\left(C^{*}(\widetilde{M})^{G}\right)$. The construction in Definition 3.1 gives rise to an invertible element

$$
\left.\left(D_{t}+S_{t}\right)\left(D_{t}-S_{t}\right)^{-1}\right|_{\Lambda^{\text {even }}\left(\widetilde{M^{\prime}}\right) \oplus \Lambda^{\text {even }}(\widetilde{M})} \in M_{2}\left(C_{L}^{*}(\widetilde{M})^{G,+}\right) .
$$


In particular, we have $D_{1}=D$ and $S_{1}=S$. Since $f$ is a controlled homotopy equivalence, it gives rise to a canonical path that connects $(D+S)(D-S)^{-1}$ to the identity operator as shown by Higson and Roe in [2]. The path is constructed out of the following path $S_{f}(t)$ connecting $S$ with $-S$,

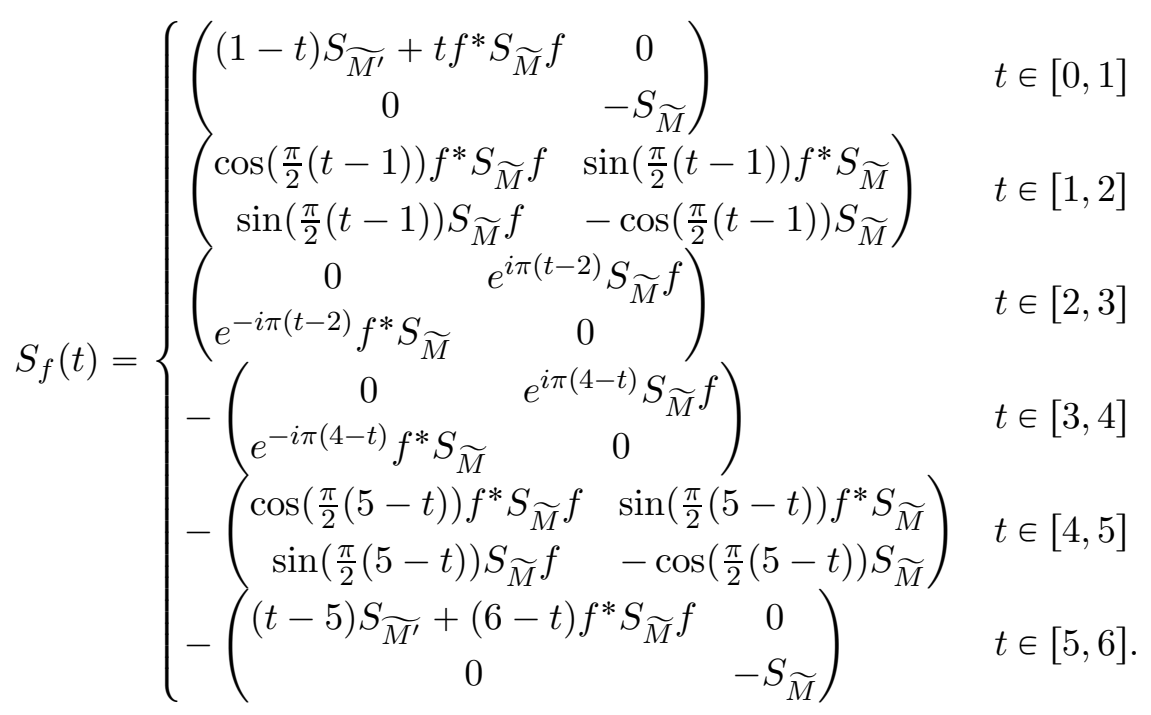

For any $t \in[0,6], S_{f}(t)$ satisfying the following conditions:

1. $D \pm S_{f}(t)$ are both invertible;

2. the invertible operator

$$
\frac{D+S}{D-S_{f}(t)}: \Lambda^{\text {even }}(\widetilde{M}) \oplus \Lambda^{\text {even }}\left(\widetilde{M}^{\prime}\right) \rightarrow \Lambda^{\text {even }}(\widetilde{M}) \oplus \Lambda^{\text {even }}\left(\widetilde{M}^{\prime}\right)
$$

belongs to $M_{2}\left(C^{*}(\widetilde{M})^{G}\right)^{+}$.

Definition 3.5. The higher rho invariant $\rho(f)$ is the $K$-theory class in $K_{1}\left(C_{L, 0}^{*}(\widetilde{M})^{G}\right)$ represented by the following invertible element

$$
\begin{cases}\left.(D+S)\left(D+S_{f}(t-1)\right)^{-1}\right|_{\Lambda^{\text {even }}\left(\widetilde{M^{\prime}}\right) \oplus \Lambda^{\text {even }}(\widetilde{M})^{\prime}}, \quad t \in[1,7], \\ \left.\left(D_{t-6}+S_{t-6}\right)\left(D_{t-6}-S_{t-6}\right)^{-1}\right|_{\Lambda^{\text {even }}\left(\widetilde{M^{\prime}}\right) \oplus \Lambda^{\text {even }}(\widetilde{M})}, \quad t \geqslant 7 .\end{cases}
$$

Even case. The even dimensional case is parallel to the odd case above. The construction in Definition 3.2 gives rise to a path of difference of projections

$$
P_{+}\left(D_{t}+S_{t}\right)-P_{+}\left(D_{t}-S_{t}\right) \in M_{2}\left(C_{L}^{*}(\widetilde{M})^{G}\right),
$$


with

$$
P_{+}\left(D_{1}+S_{1}\right)-P_{+}\left(D_{1}-S_{1}\right)=P_{+}(D+S)-P_{+}(D-S) .
$$

Let $S_{f}(t)$ be as above. Similarly, we have that:

1. $D \pm S_{f}(t)$ are both invertible;

2. $P_{+}\left(D \pm S_{f}(t)\right)$ are of finite propagation, and

$$
P_{+}(D+S)-P_{+}\left(D-S_{f}(t)\right) \in M_{2}\left(C^{*}(\widetilde{M})^{G}\right) .
$$

Thus the formal difference

$$
\left[P_{+}(D+S)\right]-\left[P_{+}\left(D-S_{f}(t)\right)\right]
$$

determines a $K$-theory class in $K_{0}\left(C^{*}(\widetilde{M})^{G}\right)$;

The higher rho invariant associated to controlled homotopy equivalence $f$ is defined as follows.

Definition 3.6. Write

$$
\begin{gathered}
\Theta_{f,+}(t)= \begin{cases}P_{+}(D+S) & t \in[1,7] \\
P_{+}\left(D_{t-6}+S_{t-6}\right) & t \geqslant 7\end{cases} \\
\Theta_{f,-}(t)= \begin{cases}P_{+}\left(D+S_{f}(t-1)\right) & t \in[1,7] \\
P_{+}\left(D_{t-6}-S_{t-6}\right) & t \geqslant 7\end{cases}
\end{gathered}
$$

As $\Theta_{f, \pm}(t)$ are projections and their difference lies in $M_{2}\left(C_{L, 0}^{*}(\widetilde{M})^{G}\right)$, the formal difference

$$
\left[\Theta_{f,+}\right]-\left[\Theta_{f,-}\right]
$$

defines a $K$-theory class $\rho(f)$ in $K_{0}\left(C_{L, 0}^{*}(\widetilde{M})^{G}\right)$, called higher rho invariant.

\section{Product formula}

In this section, we will prove the product formula for higher rho invariant associated to the signature operator for homotopy equivalence. We only consider the case for product of manifolds for now. The general case for fibered manifolds will be discussed in the next section.

Proposition 4.1. Let $M, N$ be two manifolds with dimension $m, n$ and fundamental groups $G, H$ respectively. Under the product map

$$
\left.\psi: K_{m}\left(C^{*}(\widetilde{M})^{G}\right) \otimes K_{n}\left(C^{*}(\tilde{N})^{H}\right) \rightarrow K_{m+n}(\widetilde{M} \times \tilde{N})^{G \times H}\right),
$$


we have

$$
k_{m n} \cdot \psi\left(\operatorname{Ind}\left(D_{M}^{s g n}\right) \otimes \operatorname{Ind}\left(D_{N}^{s g n}\right)\right)=\operatorname{Ind}\left(D_{M \times N}^{s g n}\right),
$$

where

$$
k_{m n}= \begin{cases}1, & m n \text { is even } \\ 2, & m n \text { is odd }\end{cases}
$$

Proof. In the following, we omit the mention of $\psi$ for simplicity. We avoid to use the fact that $S_{\widetilde{M}}^{2}=1$ throughout the proof for the purpose of further generalization. Therefore, we have to consider four cases according to the parity of both $\operatorname{dim} M$ and $\operatorname{dim} N$.

Even times odd. We first suppose that $\operatorname{dim} M$ is even and $\operatorname{dim} N$ is odd.

Write $B_{\widetilde{M} \pm}=D_{\widetilde{M}} \pm S_{\widetilde{M}}$ for short. On the product manifold $\widetilde{M} \times \widetilde{N}$, the differential operator $d_{\widetilde{M} \times \widetilde{N}}$ is given by

$$
d_{\widetilde{M} \times \widetilde{N}}=d_{\widetilde{M}} \hat{\otimes} 1+1 \hat{\otimes} d_{\widetilde{N}}=d_{\widetilde{M}} \otimes 1+E_{\widetilde{M}} \otimes d_{\widetilde{N}},
$$

where $E_{\widetilde{M}}$ is the even-odd grading operator for $\Lambda(\widetilde{M})$. Therefore

$$
D_{\widetilde{M} \times \widetilde{N}}=D_{\widetilde{M}} \otimes 1+E_{\widetilde{M}} \otimes D_{\widetilde{N}}
$$

Now we decompose $\Lambda^{\text {even }}(\widetilde{M} \times \tilde{N})$ into the direct sum of $\Lambda^{\text {even }}(\widetilde{M}) \otimes$ $\Lambda^{\text {even }}(\widetilde{N})$ and $\Lambda^{\text {odd }}(\widetilde{M}) \otimes \Lambda^{\text {odd }}(\tilde{N})$. As $\operatorname{dim} N$ is odd, the Hodge $*$-operator as well as the Poincaré duality operator $S_{\widetilde{N}}$ reverses the parity of $\Lambda(\tilde{N})$. Note that $S_{\widetilde{N}}^{2}: \Lambda^{p}(\tilde{N}) \rightarrow \Lambda^{p}(\tilde{N})$ is a multiple of identity. Therefore we identify $\Lambda(\widetilde{M}) \otimes \Lambda^{\text {odd }}(\widetilde{N})$ as $\Lambda(\widetilde{M}) \otimes \Lambda^{\text {even }}(\tilde{N})$ via $1 \otimes S_{\widetilde{N}}$. Under this identification, the higher index of signature operator is represented by the following invertible operator

$$
\begin{array}{r}
\left(B_{\widetilde{M}}^{+} \otimes 1+1 \otimes S_{\widetilde{N}} D_{\widetilde{N}}\right)\left(B_{\widetilde{M}}^{-} \otimes 1+1 \otimes S_{\widetilde{N}} D_{\widetilde{N}}\right)^{-1}: \\
\Lambda(\widetilde{M}) \otimes \Lambda^{\text {even }}(\widetilde{N}) \rightarrow \Lambda(\widetilde{M}) \otimes \Lambda^{\text {even }}(\tilde{N}) .
\end{array}
$$

Since $B_{\widetilde{M}}^{+}$is invertible, we define a path of bounded operators

$$
W_{+, s}=\frac{B_{\widetilde{M}}^{+}}{\left|B_{\widetilde{M}}^{+}\right|^{s}} \otimes 1+1 \otimes S_{\widetilde{N}} D_{\widetilde{N}}
$$

For the invertible operator $B \frac{ \pm}{\widetilde{M}}$, we denote by $P^{+}\left(B \frac{ \pm}{\widetilde{M}}\right)\left(\right.$ resp. $\left.P^{-}\left(B \frac{ \pm}{\widetilde{M}}\right)\right)$ the spectral projection of the positive (resp. negative) part of $B \frac{ \pm}{\widetilde{M}}$. We see that

$$
W_{+, 0}=B_{\widetilde{M}}^{+} \otimes 1+1 \otimes S_{\widetilde{N}} D_{\widetilde{N}}, W_{+, 1}=\left(P^{+}\left(B_{\widetilde{M}}^{+}\right)-P^{-}\left(B_{\widetilde{M}}^{+}\right)\right) \otimes 1+1 \otimes S_{\widetilde{N}} D_{\widetilde{N}}
$$


Since $D_{\widetilde{N}}$ anti-commutes with $S_{\widetilde{N}}$, we have

$$
W_{+, s}^{*} W_{+, s}=\left(B_{\widetilde{M}}^{+}\right)^{2(1-s)} \otimes 1+1 \otimes D_{\widetilde{N}}^{2}>0 .
$$

Thus $W_{+, s}$ is a path of invertible operator for every $s$ in $[0,1]$.

Similarly we define a path of invertible operator $W_{-, s}$. Thus via the homotopy $W_{+, s}\left(W_{-, s}\right)^{-1}$, the higher index of signature operator on $M \times N$ is also represented by the invertible operator

$$
W_{+, 1}\left(W_{-, 1}\right)^{-1}: \Lambda(\widetilde{M}) \otimes \Lambda^{e v e n}(\tilde{N}) \rightarrow \Lambda(\widetilde{M}) \otimes \Lambda^{\text {even }}(\tilde{N})
$$

We rewrite the expression above using $1=P^{+}\left(B \frac{ \pm}{\widetilde{M}}\right)+P^{-}\left(B \frac{ \pm}{\widetilde{M}}\right)$.

$$
\begin{aligned}
& {\left[W_{+, 1}\left(W_{-, 1}\right)^{-1}\right] } \\
= & {\left[P^{+}\left(B_{\widetilde{M}}^{+}\right) \otimes\left(S_{\widetilde{N}} D_{\widetilde{N}}+1\right)\left(S_{\widetilde{N}} D_{\widetilde{N}}-1\right)^{-1}+P^{-}\left(B_{\widetilde{M}}^{+}\right) \otimes 1\right] } \\
& -\left[P^{+}\left(B_{\widetilde{M}}^{-}\right) \otimes\left(S_{\widetilde{N}} D_{\widetilde{N}}+1\right)\left(S_{\widetilde{N}} D_{\widetilde{N}}-1\right)^{-1}+P^{-}\left(B_{\widetilde{M}}^{-}\right) \otimes 1\right] \\
= & \left(\left[P^{+}\left(B_{\widetilde{M}}^{+}\right)\right]-\left[P^{+}\left(B_{\widetilde{M}}^{-}\right)\right]\right) \times\left[\left(D_{\widetilde{N}}+S_{\widetilde{N}}\right)\left(D_{\widetilde{N}}-S_{\widetilde{N}}\right)^{-1}\right] \\
= & \operatorname{Ind}\left(D_{M}^{s g n}\right) \times \operatorname{Ind}\left(D_{N}^{s g n}\right) .
\end{aligned}
$$

The last two equalities follow from the definition of product of $K$-groups and the formula of the higher index of signature operator in Section 3.1.

Odd times even. Suppose that $M$ is odd dimensional and $N$ is even dimensional. Straightforward computation shows that

1. $S_{\widetilde{M} \times \widetilde{N}}=S_{\widetilde{M}} \otimes S_{\widetilde{N}}$ on $\Lambda(\widetilde{M}) \otimes \Lambda^{\text {even }}(\tilde{N})$,

2. $S_{\widetilde{M} \times \widetilde{N}}=-S_{\widetilde{M}} \otimes S_{\widetilde{N}}$ on $\Lambda(\widetilde{M}) \otimes \Lambda^{\text {odd }}(\tilde{N})$.

and

1. $d_{\widetilde{M} \times \tilde{N}}=d_{\widetilde{M}} \otimes 1+1 \otimes d_{\widetilde{N}}$ on $\Lambda(\widetilde{M})^{\text {odd }} \otimes \Lambda(\tilde{N})$,

2. $d_{\widetilde{M} \times \tilde{N}}=d_{\widetilde{M}} \otimes 1-1 \otimes d_{\widetilde{N}}$ on $\Lambda(\widetilde{M})^{\text {even }} \otimes \Lambda(\tilde{N})$.

Let $\Lambda_{ \pm}(\tilde{N})$ be the \pm 1 eigenspace of $S_{\widetilde{N}}$. We make the following identifications

1. Under the decomposition

$$
\begin{aligned}
& \Lambda^{\text {odd }}(\widetilde{M}) \otimes \Lambda_{+}^{\text {odd }}(\tilde{N}) \\
& \oplus \\
& \Lambda^{\text {odd }}(\widetilde{M}) \otimes \Lambda(\tilde{N})=\begin{array}{l}
\Lambda^{\text {odd }}(\widetilde{M}) \otimes \Lambda_{-}^{\text {odd }}(\tilde{N}) \\
\Lambda^{\text {odd }}(\widetilde{M}) \otimes \Lambda_{+}^{\text {even }}(\tilde{N})
\end{array} \text { and } \Lambda^{\text {odd }}(\widetilde{M} \times \widetilde{N})=\begin{array}{c}
\Lambda^{\text {even }}(\widetilde{M}) \otimes \Lambda_{-}^{\text {odd }}(\tilde{N}) \\
\Lambda^{\text {odd }}(\widetilde{M}) \otimes \Lambda_{+}^{\text {even }}(\tilde{N})
\end{array}, \\
& \Lambda^{\text {odd }}(\widetilde{M}) \otimes \Lambda_{-}^{\text {even }}(\widetilde{N}) \\
& \Lambda^{\text {even }}(\widetilde{M}) \otimes \Lambda_{+}^{\text {odd }}(\tilde{N}) \\
& \oplus \\
& \Lambda^{\text {odd }}(\widetilde{M}) \otimes \Lambda_{-}^{\text {even }}(\widetilde{N})
\end{aligned}
$$


we identify $\Lambda^{\text {odd }}(\widetilde{M}) \otimes \Lambda(\widetilde{N})$ with $\Lambda^{\text {odd }}(\widetilde{M} \times \tilde{N})$ by

$$
\left(\begin{array}{cccc}
-B_{\widetilde{M}+} \otimes 1 & & & \\
& B_{\widetilde{M}-} \otimes 1 & & \\
& & 1 \otimes 1 & \\
& & 1 \otimes 1
\end{array}\right): \Lambda^{\text {odd }}(\widetilde{M}) \otimes \Lambda(\widetilde{N}) \rightarrow \Lambda^{\text {odd }}(\widetilde{M} \times \tilde{N}) .
$$

2. Under the decomposition

$$
\begin{aligned}
& \Lambda^{\text {even }}(\widetilde{M}) \otimes \Lambda_{+}^{\text {even }}(\widetilde{N}) \quad \quad \Lambda^{\text {even }}(\widetilde{M}) \otimes \Lambda_{+}^{\text {even }}(\widetilde{N}) \\
& \Lambda^{\text {even }}(\widetilde{M} \times N)=\begin{array}{c}
\Lambda^{\text {even }}(\widetilde{M}) \otimes \Lambda_{-}^{\text {even }}(\tilde{N}) \\
\Lambda^{\text {odd }}(\widetilde{M}) \otimes \Lambda_{+}^{\text {odd }}(\tilde{N})
\end{array} \text { and } \Lambda^{\text {even }}(\widetilde{M}) \otimes \Lambda(N)=\begin{array}{c}
\Lambda^{\text {even }}(\widetilde{M}) \otimes \Lambda_{-}^{\text {even }}(\tilde{N}) \\
\Lambda^{\text {even }}(\widetilde{M}) \otimes \Lambda_{+}^{\text {odd }}(\tilde{N})
\end{array}, \\
& \Lambda^{\text {odd }}(\widetilde{M}) \otimes \Lambda_{-}^{\text {odd }}(\tilde{N}) \\
& \Lambda^{\text {even }}(\widetilde{M}) \otimes \Lambda_{-}^{\text {odd }}(\widetilde{N})
\end{aligned}
$$

we identify $\Lambda^{\text {even }}(\widetilde{M} \times \widetilde{N})$ with $\Lambda^{\text {even }}(\widetilde{M}) \otimes \Lambda(\widetilde{N})$ by

$$
\left(\begin{array}{cccc}
1 \otimes 1 & & & \\
& -1 \otimes 1 & & \\
& & -B_{\widetilde{M}-} \otimes 1 & \\
& & & -B_{\widetilde{M}+} \otimes 1
\end{array}\right): \Lambda^{e v e n}(\widetilde{M} \times \tilde{N}) \rightarrow \Lambda^{e v e n}(\widetilde{M}) \otimes \Lambda(\tilde{N}) .
$$

With these identifications, we have

$$
d_{\widetilde{M} \times \widetilde{N}}+d_{\widetilde{M} \times \widetilde{N}}^{*}+S_{\widetilde{M} \times \widetilde{N}}=\left\{\begin{array}{cc}
B_{\widetilde{M}+} \otimes 1+B_{\widetilde{M}+} \otimes D_{\widetilde{N}} & \text { on } \Lambda^{\text {odd }}(\widetilde{M}) \otimes \Lambda_{+}^{\text {even }}(\tilde{N}) \\
B_{\widetilde{M}-}^{2} B_{\widetilde{M}+} \otimes 1+B_{\widetilde{M}+} \otimes D_{\widetilde{N}} & \text { on } \Lambda^{\text {odd }}(\widetilde{M}) \otimes \Lambda_{+}^{\text {odd }}(\tilde{N}) \\
-B_{\widetilde{M}-} \otimes 1+B_{\widetilde{M}-} \otimes D_{\widetilde{N}} & \text { on } \Lambda^{\text {odd }}(\widetilde{M}) \otimes \Lambda_{-}^{\text {even }}(\tilde{N}) \\
-B_{\widetilde{M}+}^{2} B_{\widetilde{M}-} \otimes 1+B_{\widetilde{M}-} \otimes D_{\widetilde{N}} & \text { on } \Lambda^{\text {odd }}(\widetilde{M}) \otimes \Lambda_{+}^{\text {odd }}(\tilde{N})
\end{array}\right.
$$

Note that $B_{\widetilde{M} \pm}^{2}$ are positive invertible operators. It follows that $d_{\widetilde{M} \times \widetilde{N}}+$ $d_{\widetilde{M} \times \tilde{N}}^{*}+S_{\widetilde{M} \times \widetilde{N}}$ is homotopic to

$$
\left(\begin{array}{cc}
B_{\widetilde{M}+} & 0 \\
0 & B_{\widetilde{M}-}
\end{array}\right) S_{\widetilde{N}}+\left(\begin{array}{cc}
B_{\widetilde{M}-} & 0 \\
0 & B_{\widetilde{M}+}
\end{array}\right) D_{\widetilde{N}}: \Lambda^{\text {odd }}(\widetilde{M}) \otimes \Lambda(\widetilde{N}) \rightarrow \Lambda^{\text {even }}(\widetilde{M}) \otimes \Lambda(\widetilde{N})
$$

where the matrix form is written with respect to the decomposition

$$
\Lambda(\tilde{N})=\Lambda_{+}(\tilde{N}) \oplus \Lambda_{-}(\tilde{N}) .
$$


Since $D_{\widetilde{N}}$ is off-diagonal, $d_{\widetilde{M} \times \widetilde{N}}+d_{\widetilde{M} \times \widetilde{N}}^{*}+S_{\widetilde{M} \times \widetilde{N}}$ is in turn homotopic to

$$
V=\left(\begin{array}{cc}
B_{\widetilde{M}+} & 0 \\
0 & B_{\widetilde{M}-}
\end{array}\right) S_{\widetilde{N}} f\left(D_{\widetilde{N}}\right)+\left(\begin{array}{cc}
B_{\widetilde{M}-} & 0 \\
0 & B_{\widetilde{M}+}
\end{array}\right) g\left(D_{\widetilde{N}}\right)
$$

where $g(x)=\frac{x}{\sqrt{1+x^{2}}}$ and $f(x)=\frac{1}{\sqrt{1+x^{2}}}$. In the meantime, $d_{\widetilde{M} \times \widetilde{N}}+d_{\widetilde{M} \times \widetilde{N}}^{*}-$ $S_{\widetilde{M} \times \widetilde{N}}$ is homotopic to

$$
U=\left(\begin{array}{cc}
B_{\widetilde{M}-} & 0 \\
0 & B_{\widetilde{M}+}
\end{array}\right) S_{\widetilde{N}} f\left(D_{\widetilde{N}}\right)+\left(\begin{array}{cc}
B_{\widetilde{M}-} & 0 \\
0 & B_{\widetilde{M}+}
\end{array}\right) g\left(D_{\widetilde{N}}\right)
$$

It follows that

$$
\begin{gathered}
V U^{-1}: \Lambda^{e v e n}(\widetilde{M} \times \widetilde{N}) \rightarrow \Lambda^{e v e n}(\widetilde{M} \times \widetilde{N}) \\
V U^{-1}=\left[\left(d_{\widetilde{M}}+d_{\widetilde{M}}^{*}\right) \otimes 1+S_{\widetilde{M}} \otimes S_{2} S_{1} S_{2}\right]\left(\begin{array}{ll}
B_{\widetilde{M}-}^{-1} & \\
& B_{\widetilde{M}+}^{-1}
\end{array}\right),
\end{gathered}
$$

where

$$
S_{1}=S_{\tilde{N}}, \text { and } S_{2}=g\left(D_{\tilde{N}}\right)+S_{\tilde{N}} f\left(D_{\tilde{N}}\right) .
$$

Note that $S_{2} S_{1} S_{2}$ is a symmetry, i.e. $S_{2} S_{1} S_{2}$ can be approximated by finite propagation operators, and $\left(S_{2} S_{1} S_{2}\right)^{2}-1$ belongs to $C^{*}(\tilde{N})^{H}$. We define

$$
P=\frac{S_{2} S_{1} S_{2}+1}{2}
$$

Now one can see that the higher index of $D_{M \times N}^{s g n}$ is actually represented by

$$
\begin{aligned}
& {\left[\left(d_{\widetilde{M}}+d_{\widetilde{M}}^{*}\right) \otimes 1+S_{\widetilde{M}} \otimes S_{2} S_{1} S_{2}\right]\left(\begin{array}{cc}
B_{\widetilde{M}-}^{-1} & 0 \\
0 & B_{\widetilde{M}+}^{-1}
\end{array}\right) } \\
= & {\left[B_{\widetilde{M}+} \otimes P+B_{\widetilde{M}-} \otimes(1-P)\right]\left(\left(\begin{array}{cc}
B_{\widetilde{M}-}^{-1} & 0 \\
0 & 0
\end{array}\right)+\left(\begin{array}{cc}
0 & 0 \\
0 & B_{\widetilde{M}+}^{-1}
\end{array}\right)\right) } \\
= & \left(\begin{array}{cc}
B_{\widetilde{M}+} B_{\widetilde{M}-}^{-1} & 0 \\
0 & 1
\end{array}\right) \otimes P+\left(\begin{array}{cc}
1 & 0 \\
0 & B_{\widetilde{M}-} B_{\widetilde{M}+}^{-1}
\end{array}\right) \otimes(1-P) \\
= & \left(\left(\begin{array}{cc}
B_{\widetilde{M}+} B_{\widetilde{M}-}^{-1} & 0 \\
0 & B_{\widetilde{M}+} B_{\widetilde{M}-1}^{-1}
\end{array}\right) \otimes P+\left(\begin{array}{ll}
1 & 0 \\
0 & 1
\end{array}\right) \otimes(1-P)\right)\left(\begin{array}{cc}
1 & 0 \\
0 & B_{\widetilde{M}-} B_{\widetilde{M}+}^{-1}
\end{array}\right) \\
= & B_{\widetilde{M}+} B_{\widetilde{M}-}^{-1} \otimes\left([P]-\left[\left(\begin{array}{ll}
0 & 0 \\
0 & 1
\end{array}\right)\right]\right),
\end{aligned}
$$

which is equal to $\operatorname{Ind}\left(D_{M}^{s g n}\right) \otimes \operatorname{Ind}\left(D_{N}^{s g n}\right)$ since

$$
[P]-\left[\left(\begin{array}{cc}
0 & 0 \\
0 & 1
\end{array}\right)\right]=\left[P_{+}\left(D_{\widetilde{N}}+S_{\widetilde{N}}\right)\right]-\left[P_{+}\left(D_{\widetilde{N}}-S_{\widetilde{N}}\right)\right]
$$

as computed in [3]. 
Even times even. Suppose that both $M$ and $N$ are even dimensional. In [13], it is shown that

$$
\begin{aligned}
\operatorname{Ind}\left(D_{N}^{s g n}\right) \otimes \operatorname{Ind}\left(D_{\mathbb{R}}^{s g n}\right) & =\operatorname{Ind}\left(D_{N \times \mathbb{R}}^{s g n},\right. \\
\operatorname{Ind}\left(D_{M \times N}^{s g n}\right) \otimes \operatorname{Ind}\left(D_{\mathbb{R}}^{s g n}\right) & =\operatorname{Ind}\left(D_{M \times N \times \mathbb{R}}^{s g n} .\right.
\end{aligned}
$$

Note that $\operatorname{Ind}\left(D_{\mathbb{R}}^{s g n}\right)$ is the generator of

$$
K_{1}\left(C^{*}(\mathbb{R})\right) \cong K_{1}\left(C_{L}^{*}(\mathbb{R})\right) \cong \mathbb{Z}
$$

Now we have

$$
\begin{aligned}
& \left.\operatorname{Ind}\left(D_{M}^{s g n}\right)\right) \otimes \operatorname{Ind}\left(D_{N}^{s g n}\right) \otimes \operatorname{Ind}\left(D_{\mathbb{R}}^{s g n}\right) \\
= & \left.\operatorname{Ind}\left(D_{M}^{s g n}\right)\right) \otimes \operatorname{Ind}\left(D_{N \times \mathbb{R}}^{s g n}\right) \\
= & \operatorname{Ind}\left(D_{M \times N \times \mathbb{R}}^{s g n}\right) \\
= & \operatorname{Ind}\left(D_{M \times N}^{s g n}\right) \otimes \operatorname{Ind}\left(D_{\mathbb{R}}^{s g n}\right) .
\end{aligned}
$$

It follows that

$$
\operatorname{Ind}\left(D_{M}^{s g n}\right) \otimes \operatorname{Ind}\left(D_{N}^{s g n}\right)=\operatorname{Ind}\left(D_{M \times N}^{s g n}\right) .
$$

Odd times odd. Let $M$ and $N$ be both odd dimensional manifolds. In this case, as shown in [13], we have

$$
\begin{aligned}
2 \operatorname{Ind}\left(D_{N}^{s g n}\right) \otimes \operatorname{Ind}\left(D_{\mathbb{R}}^{s g n}\right) & =\operatorname{Ind}\left(D_{N \times \mathbb{R}}^{s g n}\right), \\
\operatorname{Ind}\left(D_{M \times N}^{s g n}\right) \otimes \operatorname{Ind}\left(D_{\mathbb{R}}^{s g n}\right) & =\operatorname{Ind}\left(D_{M \times N \times \mathbb{R}}^{s g n}\right) .
\end{aligned}
$$

Now we have

$$
\begin{aligned}
& \left.2 \operatorname{Ind}\left(D_{M}^{s g n}\right)\right) \otimes \operatorname{Ind}\left(D_{N}^{s g n}\right) \otimes \operatorname{Ind}\left(D_{\mathbb{R}}^{s g n}\right) \\
= & \operatorname{Ind}\left(D_{M}^{s g n}\right) \otimes \operatorname{Ind}\left(D_{N \times \mathbb{R}}^{s g n}\right) \\
= & \operatorname{Ind}\left(D_{M \times N \times \mathbb{R}}^{s g n}\right) \\
= & \operatorname{Ind}\left(D_{M \times N}^{s g n}\right) \otimes \operatorname{Ind}\left(D_{\mathbb{R}}^{s g n}\right) .
\end{aligned}
$$

It follows that

$$
2 \operatorname{Ind}\left(D_{M}^{s g n}\right) \otimes \operatorname{Ind}\left(D_{N}^{s g n}\right)=\operatorname{Ind}\left(D_{M \times N}^{s g n}\right)
$$

Note that in the proof of Proposition of 4.1, we actually do not demand that $S_{\widetilde{M}}^{2}=1$. It follows that given the definition of $K$-homology class of signature and higher rho invariant, the argument above can be easily generalized to show the following Proposition and Theorem: 
Proposition 4.2. With the same notations, under the product map

$$
\psi_{L}: K_{m}\left(C_{L}^{*}(\widetilde{M})^{G}\right) \otimes K_{n}\left(C_{L}^{*}(\tilde{N})^{H}\right) \rightarrow K_{m+n}\left(C_{L}^{*}(\widetilde{M} \times \tilde{N})^{G \times H}\right),
$$

there is a product formula of $K$-homology class of signature operator on $M$ and $N$ goes as follows,

$$
k_{m n} \cdot \psi_{L}\left(\left[D_{M}^{s g n}\right] \otimes\left[D_{N}^{s g n}\right]\right)=\left[D_{M \times N}^{s g n}\right]
$$

where

$$
k_{m n}=\left\{\begin{array}{l}
1, \quad m n \text { is even }, \\
2, \quad m n \text { is odd } .
\end{array}\right.
$$

Theorem 4.3. Suppose that $M^{\prime}, M$ are two closed oriented Riemannian manifolds and $f: M^{\prime} \rightarrow M$ is a homotopy equivalence. Write $m=\operatorname{dim} M^{\prime}=$ $\operatorname{dim} M$. Let $\widetilde{M^{\prime}}, \widetilde{M}$ be theirs Galois $G$-covering spaces respectively. Under the product map

$$
\psi_{L, 0}: K_{m}\left(C_{L}^{*}(\widetilde{M})^{G}\right) \otimes K_{n}\left(C_{L, 0}^{*}(\tilde{N})^{H}\right) \rightarrow K_{m+n}\left(C_{L, 0}^{*}(\widetilde{M} \times \tilde{N})^{G \times H}\right),
$$

there is a product formula goes as follows,

$$
k_{m n} \cdot \psi_{L, 0}\left(\rho(f) \otimes\left[D_{N}^{s g n}\right]\right)=\rho\left(f \times I_{N}\right),
$$

where $I_{N}: N \rightarrow N$ is the identity map, and

$$
k_{m n}=\left\{\begin{array}{l}
1, \quad m n \text { is even } \\
2, \quad m n \text { is odd }
\end{array}\right.
$$

\section{Product formula for fibered manifolds}

In this section, we generalize the product formula given in the previous section to fibered manifolds. We will first introduce a series of family geometric $C^{*}$ algebras with respect to the fibration. Next we define the family version of $K$-homology class and higher rho invariant of fiberwise signature operator in $K$-theory of these $C^{*}$-algebras. At last, We will prove Theorem 1.1 and 1.2.

\subsection{Family algebras}

In this subsection, we introduce family geometric $C^{*}$-algebras associated to a fibered manifold.

Let $\pi: E \rightarrow B$ be a fibration with fiber $F$ and base space $B$. Assume that $E, F$ and $B$ are closed connected oriented Riemannian manifolds. The fibration induces a long exact sequence of homotopy groups

$$
\cdots \longrightarrow \pi_{2}(B) \stackrel{\partial}{\longrightarrow} \pi_{1}(F) \longrightarrow \pi_{1}(E) \stackrel{\pi_{*}}{\longrightarrow} \pi_{1}(B) \longrightarrow 0
$$


Denote by $\widetilde{E}$ and $\widetilde{B}$ the universal covering of $E$ and $B$. From the exactness of the above sequence, we see that $\partial\left(\pi_{2}(B)\right)$ is a normal subgroup of $\pi_{1}(F)$. Write $\Gamma=\pi_{1}(F) / \partial\left(\pi_{2}(B)\right)$. The above exact sequence shows that $\widetilde{E}$ is also a fibration on $\widetilde{B}$ with fiber projection $\widetilde{\pi}: \widetilde{E} \rightarrow \widetilde{B}$ and fiber $\widetilde{F}$, the Galois $\Gamma$-covering of $F$.

From now on, we will write $G=\pi_{1}(E)$ and $H=\pi_{1}(B)$ for short. Recall that the equivariant Roe algebras $C^{*}(\widetilde{E})^{G}$ is defined to be the completion of $G$ equivariant, locally compact operators with finite propagation as in Definition 2.2. Now let us define the equivariant family Roe algebra.

First we construct an equivariant Roe algebra bundle over $B$. View the fiber bundle $E$ over $B$ as gluing many pieces of local trivialization by a series of diffeomorphisms of $F$. More precisely, there exists an open cover $\left\{V_{\alpha}\right\}$ of $B$ and continuous maps $\varphi_{\alpha \beta}: V_{\alpha} \cap V_{\beta} \rightarrow \operatorname{Diff}(F)$ such that the fiber bundle $E$ is equivalent to the tuple $\left(V_{\alpha} \times F, \varphi_{\alpha \beta}\right)$, that is, every continuous section $s$ of $E$ is equivalent to a series of continuous maps $s_{\alpha}: V_{\alpha} \rightarrow F$ satisfying the cocycle condition, $\varphi_{\alpha \beta}(x) s_{\alpha}(x)=s_{\beta}(x)$ for any $x \in V_{\alpha} \cap V_{\beta}$.

By our previous arguments, when turning to the universal covering, $\widetilde{E}$ is also an $\widetilde{F}$-bundle over $\widetilde{B}$. Assume that every open set in $\left\{V_{\alpha}\right\}$ is small enough so that it lifts to a $\pi_{1}(B)$-equivariant open cover $\left\{U_{j}\right\}$ of $B$, each open set of which is homeomorphic to the Euclidean space and trivialize $\widetilde{E}$. Also, the transition map lifts to $\left\{\psi_{i j}\right\}$ with $\psi_{i j}: U_{i} \cap U_{j} \rightarrow \operatorname{Diff}(\widetilde{F})$. Recall the subspace $\mathbb{C}[\tilde{F}]^{\Gamma}$ of the equivariant Roe algebra $C^{*}(\widetilde{F})^{\Gamma}$ as in Definition 2.4. For any $x \in U_{i} \cap U_{j}, \psi_{i j}(x)$ induces an isomorphism $\psi_{i j, *}(x)$ of $C^{*}(\widetilde{F})^{\Gamma}$ by conjugation, which maps $\mathbb{C}[\widetilde{F}]^{\Gamma}$ to itself. This induces the following fiber bundle.

Definition 5.1 (Equivariant family Roe algebra). Recall that $G=\pi_{1}(E)$ and $\Gamma=\pi_{1}(F) / \partial\left(\pi_{2}(B)\right)$. A continuous section of the fiber bundle given by $\left(\left\{U_{i}\right\},\left\{\psi_{i j, *}\right\}\right)$ is defined by a series of norm-continuous maps $s_{i}: U_{i} \rightarrow C^{*}(\widetilde{F})^{\Gamma}$ satisfying the cocycle condition, $\psi_{i j, *}(x) s_{i}(x)=s_{j}(x)$ for any $x \in U_{i} \cap U_{j}$. Let $\mathbb{C}[\widetilde{E}, \widetilde{B}]^{G}$ be the collection of uniformly norm-bounded and uniformly normcontinuous sections that are invariant under $\pi_{1}(B)$-action, and have uniformly finite propagation on $\widetilde{B}$. The norm of such a section $\left\{s_{j}\right\}$ is defined to be $\sup _{j} \sup _{x \in U_{j}}\left\|s_{j}(x)\right\|$. Denote the completion of $\mathbb{C}[\widetilde{E}, \widetilde{B}]^{G}$ by $C^{*}(\widetilde{E}, \widetilde{B})^{G}$.

We mentioned that Definition 5.1 is related to the "Groupoid Roe algebra" given by Tang, Willett and Yao ( [10, Definition 3.6]).

It is easy to verify that the above definition is independent of the local trivialization. Similarly, we define the corresponding equivariant family localization algebra.

Definition 5.2 (Equivariant family localization and obstruction algebras). The equivariant family localization algebra $C_{L}^{*}(\widetilde{E}, \widetilde{B})^{G}$ is the completion of 
uniformly norm-bounded and uniformly norm-continuous paths $s:[0,+\infty) \rightarrow$ $C^{*}(\widetilde{E}, \widetilde{B})^{G}$ such that the propagation of $s(t)$ goes to zero as $t$ goes to infinity uniformly on $\widetilde{B}$, where the norm of $s(t)$ is defined to be $\sup _{t \in[0,+\infty)}\|s(t)\|$. The equivariant family obstruction algebra $C_{L, 0}^{*}(\widetilde{E}, \widetilde{B})^{G}$ is then defined to be the kernel of the family assembly map:

$$
\begin{aligned}
\mathrm{ev}: C_{L}^{*}(\widetilde{E}, \widetilde{B})^{G} & \rightarrow C^{*}(\widetilde{E}, \widetilde{B})^{G} \\
s & \mapsto s(1) .
\end{aligned}
$$

\subsection{Product map of $K$-theory}

In this subsection, we construct the productive map on the family $C^{*}$-algebras.

Theorem 5.3. Recall that $G=\pi_{1}(E)$ and $H=\pi_{1}(B)$. There are product maps

$$
\begin{aligned}
\phi: K_{m}\left(C_{L}^{*}(\widetilde{B})^{H}\right) \times K_{n}\left(C_{L}^{*}(\widetilde{E}, \widetilde{B})^{G}\right) & \rightarrow K_{m+n}\left(C_{L}^{*}(\widetilde{E})^{G}\right) \\
\phi_{0}: K_{m}\left(C_{L}^{*}(\widetilde{B})^{H}\right) \times K_{n}\left(C_{L, 0}^{*}(\widetilde{E}, \widetilde{B})^{G}\right) & \rightarrow K_{m+n}\left(C_{L, 0}^{*}(\widetilde{E})^{G}\right)
\end{aligned}
$$

that generalize the maps defined in (2.3).

Proof. Without loss of generality, we assume that both $m$ and $n$ are even. We will only give in details the construction of

$$
\phi_{0}: K_{0}\left(C_{L}^{*}(\widetilde{B})^{H}\right) \times K_{0}\left(C_{L, 0}^{*}(\widetilde{E}, \widetilde{B})^{G}\right) \rightarrow K_{0}\left(C_{L, 0}^{*}(\widetilde{E})^{G}\right) .
$$

Suppose that $f_{t} \in\left(C_{L}^{*}(\widetilde{B})^{H}\right)^{+}$represents a $K_{0}$-class, which has finite propagation that goes to zero as $t$ goes to infinity, and is a $1 / 10$-projection, that is, $f_{t}^{*}=f_{t}$ and $\left\|f_{t}^{2}-f_{t}\right\|<1 / 10$. Similarly, we suppose that $g_{t} \in\left(C_{L, 0}^{*}(\widetilde{E}, \widetilde{B})^{G}\right)^{+}$ is a 1/10-projection, has finite propagation that goes to zero uniformly as $t$ goes to infinity, and satisfies that $g_{1}=1$. Furthermore, we assume that $f_{t}-1$ and $g_{t}-1$ are given by kernel operators acting on $L^{2}$-sections as in Definition 2.4 .

Choose $r>0$ small enough such that for any $x \in B$, the restriction of the fiber bundle $E$ to the $r$-ball near $x$ is trivial. Since $f_{t}$ and $f_{t+M}$ are homotopic for any $M>0$, we may assume that the propagation of $f_{t}$ is smaller than $r$. By the local triviality, we define

$$
\phi\left(\left[f_{t}\right] \otimes\left[g_{t}\right]\right)=\left[\left(f_{t}-1\right) \otimes\left(g_{t}-1\right)+1\right],
$$

where $\left(f_{t}-1\right) \otimes\left(g_{t}-1\right)+1 \in\left(C_{L, 0}^{*}(\widetilde{E})^{G}\right)^{+}$is given by

$$
\begin{aligned}
& \left(\left(\left(f_{t}-1\right) \otimes\left(g_{t}-1\right)\right) h\right)(x, y) \\
= & \int_{\widetilde{B}} \int_{\widetilde{F}}\left(f_{t}-1\right)\left(x, x^{\prime}\right) \otimes\left(g_{t}-1\right)_{x^{\prime}}\left(y, y^{\prime}\right) h\left(x^{\prime}, y^{\prime}\right) d y^{\prime} d x^{\prime},
\end{aligned}
$$


with $h \in L^{2}(\widetilde{E})$. The above expression makes sense as the propagation of $f_{t}$ is small enough. It is easy to verify that $\left(f_{t}-1\right) \otimes\left(g_{t}-1\right)+1$ is at most a $3 / 10$-projection, which gives rise to a $K_{0}$-class.

Now passing to the matrix algebra and the Grothendieck group, we obtain the product map.

\subsection{Family higher invariants}

In this subsection, we introduce the family version of higher invariants of the signature operator on fibered manifold, and prove Theorem 1.1 and 1.2.

On the fibered manifold $E$, the vertical differentials and Poincaré duality are well-defined as they are compatible with the transition maps. Thus the family $K$-homology class of the vertical signature operator $\left[D_{E, B}^{s g n}\right] \in K_{\operatorname{dim} F}\left(C_{L}^{*}(\widetilde{E}, \widetilde{B})^{G}\right)$ is defined similarly as in Definition 3.1 and 3.2.

Theorem 5.4. We have the following product formula for family K-homology class of family signature operator along $F$ holds:

$$
k_{B, F} \cdot \phi\left(\left[D_{B}^{s g n}\right] \otimes\left[D_{E, B}^{s g n}\right]\right)=\left[D_{E}^{s g n}\right],
$$

where $k_{B, F}=1$ when $\operatorname{dim} B \cdot \operatorname{dim} F$ is even, and $k_{B, F}=2$ otherwise, and $\phi$ is the product map

$$
\phi: K_{\operatorname{dim} B}\left(C_{L}^{*}(\widetilde{B})^{H}\right) \otimes K_{\operatorname{dim} F}\left(C_{L}^{*}(\widetilde{E}, \widetilde{B})^{G}\right) \rightarrow K_{\operatorname{dim} E}\left(C_{L}^{*}(\widetilde{E})^{G}\right) .
$$

We shall also define the family higher rho invariant of a fiberwise homotopy equivalence. Suppose we have two fibrations over the same base

$$
F^{\prime} \longrightarrow E^{\prime} \stackrel{\pi^{\prime}}{\longrightarrow} B \text { and } F \longrightarrow E \stackrel{\pi}{\longrightarrow} B
$$

Let $f: E^{\prime} \rightarrow E$ be a fiberwise homotopy equivalence, that is, the following diagram commutes

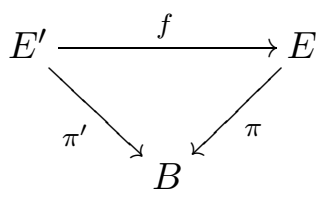

as well as replacing $f$ with its homotopy inverse and the corresponding homotopy. Using the vertical differential and the Poincare duality operator, we define a family higher rho invariant $\rho(f ; B) \in K_{\operatorname{dim} F}\left(C_{L, 0}^{*}(\widetilde{E}, \widetilde{B})^{G}\right)$ as in Definition 3.5. 
Theorem 5.5. With the same notation as above, we have the following product formula for family higher rho invariant associated to fiberwise homotopy equivalence holds:

$$
k_{B, F} \phi\left(\left[D_{B}^{s g n}\right] \otimes \rho(f ; B)\right)=\rho(f),
$$

where $k_{B, F}=1$ when $\operatorname{dim} B \cdot \operatorname{dim} F$ is even, and $k_{B, F}=2$ otherwise, and $\phi_{0}$ is the product map

$$
\phi_{0}: K_{\operatorname{dim} B}\left(C_{L}^{*}(\widetilde{B})_{r}^{H}\right) \otimes K_{\operatorname{dim} F}\left(C_{L, 0}^{*}(\widetilde{E}, \widetilde{B})^{G}\right) \rightarrow K_{\operatorname{dim} E}\left(C_{L, 0}^{*}(\widetilde{E})^{G}\right) .
$$

In the following, we only prove Theorem 5.5 in details. The proof for Theorem 5.4 is similar.

We need some definitions to prepare for the proof of Theorem 5.5.

Definition 5.6. For any element $T \in C^{*}(\widetilde{E})^{G}$, we define the propagation of $T$ along the base space $\widetilde{B}$ by

$$
\operatorname{prop}_{\widetilde{B}}(T)=\sup \{d(\tilde{\pi}(x), \tilde{\pi}(y)):(x, y) \in \operatorname{Supp}(T)\},
$$

where $\widetilde{\pi}$ is the lift of the fiber projection $\pi: E \rightarrow B$.

We need the following $C^{*}$-algebra generated by elements in $C_{L, 0}^{*}(\widetilde{E})^{G}$ that can be localized horizontally. This is a generalization of the equivariant localization algebra defined in Definition 2.2.

Definition 5.7. Define $C_{\widetilde{B}, L, 0}^{*}(\widetilde{E})^{G}$ to be the $C^{*}$-algebra generated by paths $f:[1,+\infty) \rightarrow C_{L, 0}^{*}(\widetilde{E})^{G}$ such that $f(s)$ is uniformly norm-continuous and uniformly norm-bounded, and its propagation along $\widetilde{B}$ is finite and goes to zero uniformly as $s$ goes to $\infty$. The norm of $f \in C_{\widetilde{B}, L, 0}^{*}(\widetilde{E})^{G}$ is given by the supreme of its norm in $C_{L, 0}^{*}(\widetilde{E})^{G}$, that is, $\|f\|=\sup _{s \geqslant 1}\|f(s)\|$.

There is an evaluation map

$$
\text { ev : } C_{\widetilde{B}, L, 0}^{*}(\widetilde{E})^{G} \rightarrow C_{L, 0}^{*}(\widetilde{E})^{G},
$$

which induces a $K$-theoretical map denoted by $\mathrm{ev}_{*}$.

If $X$ is a closed Riemannian manifold, the equivariant localization algebra $C_{L}^{*}(\tilde{X})^{\pi_{1} X}$ admits a Mayer-Vietoris sequence for a partition of $X$. More precisely, if $U_{1}, U_{2}$ are two open sets on $X$ and $\widetilde{U_{1}}, \widetilde{U_{2}}$ are their lifts to $\tilde{X}$, then 
we have the following six-term exact sequence (cf: [16, Proposition 3.11]).

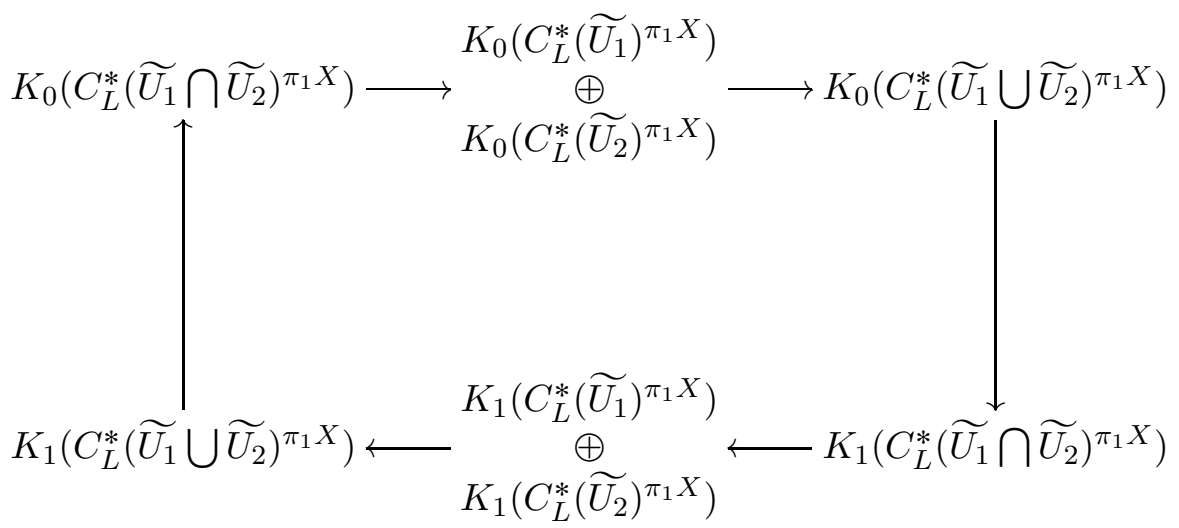

As the $C^{*}$-algebra $C_{\widetilde{B}, L, 0}^{*}(\widetilde{E})^{G}$ is generated by elements that can be localized along $B$, it also admits a Mayer-Vietoris sequence as above for two open sets on the base space.

Proposition 5.8. Let $U_{1}, U_{2}$ be two open sets on $B$ and $\widetilde{U_{1}}, \widetilde{U_{2}}$ their lifts to $\widetilde{B}$. Let $\widetilde{E}_{\widetilde{U}_{1}}$ and $\widetilde{E}_{\widetilde{U}_{2}}$ be the restriction of $\widetilde{E}$ to $\widetilde{U_{1}}$ and $\widetilde{U_{2}}$ respectively. We have the following six-term exact sequence:

$$
\begin{aligned}
& K_{0}\left(C_{\widetilde{B}, L, 0}^{*}\left(\widetilde{E}_{\widetilde{U_{1}}} \cap \widetilde{E}_{\widetilde{U_{2}}}\right)^{G}\right) \longrightarrow \underset{K_{0}\left(C_{\widetilde{B}, L, 0}^{*}\left(\widetilde{E}_{\widetilde{U_{2}}}\right)^{G}\right)}{K_{0}\left(C_{\widetilde{B}, L, 0}^{*}\left(\widetilde{E}_{\widetilde{U_{1}}}\right)^{G}\right)} \longrightarrow K_{0}\left(C_{\widetilde{B}, L, 0}^{*}\left(\widetilde{E}_{\widetilde{U_{1}}} \cup \widetilde{E}_{\widetilde{U_{2}}}\right)^{G}\right) \\
& \left.K_{1}\left(C_{\widetilde{B}, L, 0}^{*}\left(\widetilde{E}_{\widetilde{U_{1}}} \cup \widetilde{E}_{\widetilde{U}_{2}}\right)^{G}\right) \longleftarrow C_{\widetilde{B}, L, 0}^{*}\left(\widetilde{E}_{\widetilde{U_{2}}}\right)^{G}\right)
\end{aligned}
$$

Proof. We sketch the proof of Proposition 5.8 as follows, which is essentially the same as the proof of Proposition 3.11 in [16]. For any open subset $Y$ of $B$, we define $\left(C_{\widetilde{B}, L, 0}^{*}(\widetilde{E})^{G}\right)_{Y}$ to be the $C^{*}$-subalgebra of $C_{\widetilde{B}, L, 0}^{*}(\widetilde{E})^{G}$ generated by all paths $f:[1, \infty) \rightarrow C_{L, 0}^{*}(\widetilde{E})^{G}$ such that for any $s, t \in[1, \infty), \operatorname{prop}(f(s, t))<$ $\infty$ as an operator in $\mathbb{C}[\widetilde{E}]^{G}$ and for any $\varepsilon>0$, there exists $S>0$ for any $s>S$ and $t \in[1, \infty), \operatorname{Supp}(f(s, t))$ lies in the $\varepsilon$-neighborhood of $\widetilde{E}_{\widetilde{Y}} \times \widetilde{E}_{\widetilde{Y}}$, where $\widetilde{E}_{\widetilde{Y}}$ is the restriction of $\widetilde{E}$ to $Y$. 
There is a natural inclusion $i: C_{\widetilde{B}, L, 0}^{*}\left(\widetilde{E}_{\widetilde{Y}}\right)^{G} \rightarrow\left(C_{\widetilde{B}, L, 0}^{*}(\widetilde{E})^{G}\right)_{Y}$. For any $f \in\left(C_{\widetilde{B}, L, 0}^{*}(\widetilde{E})^{G}\right)_{Y}$, we have a natural homotopy between $f$ and $f_{s_{0}}(s, t)=$ $f\left(s+s_{0}, t\right)$, whose support is closed to $\widetilde{E}_{\widetilde{Y}} \times \widetilde{E}_{\widetilde{Y}}$. This shows that for any $\delta>0$, any $K$-theory element of $\left(C_{\widetilde{B}, L, 0}^{*}(\widetilde{E})^{G}\right)_{Y}$ admits a representative whose support lies in $\widetilde{E}_{\widetilde{Y}_{\delta}} \times \widetilde{E}_{\widetilde{Y}_{\delta}}$, where $Y_{\delta}$ is the $\delta$-neighborhood of $Y$. As an analogue of [16, Proposition 3.7], we see that $C_{\widetilde{B}, L, 0}^{*}\left(\widetilde{E}_{\widetilde{Y}}\right)^{G}$ and $C_{\widetilde{B}, L, 0}^{*}\left(\widetilde{E}_{\widetilde{Y}_{\delta}}\right)^{G}$ are isomorphic on $K$-theoretical level for small $\delta$. This shows that the $K$-theoretical map $i_{*}$ is surjective. The injectivity of $i_{*}$ goes similarly.

Note that $\left(C_{\widetilde{B}, L, 0}^{*}(\widetilde{E})^{G}\right)_{U_{1}}$ and $\left(C_{\widetilde{B}, L, 0}^{*}(\widetilde{E})^{G}\right)_{U_{2}}$ are closed ideals of $C_{\widetilde{B}, L, 0}^{*}(\widetilde{E})^{G}$. And we also have that

$$
\left(C_{\widetilde{B}, L, 0}^{*}(\widetilde{E})^{G}\right)_{U_{1}}+\left(C_{\widetilde{B}, L, 0}^{*}(\widetilde{E})^{G}\right)_{U_{2}}=\left(C_{\widetilde{B}, L, 0}^{*}(\widetilde{E})^{G}\right)_{U_{1}}
$$

Now the proposition follows from the $K$-theoretical six-term exact sequence (cf: [5, Lemma 3.1]).

In the following Lemma, we show that there exists a natural map

$$
\phi_{L, 0}: K_{m}\left(C_{L}^{*}(\widetilde{B})^{H}\right) \otimes K_{n}\left(C_{L, 0}^{*}(\widetilde{E}, \widetilde{B})^{G}\right) \longrightarrow K_{m+n}\left(C_{\widetilde{B}, L, 0}^{*}(\widetilde{E})^{G}\right)
$$

compatible with $\phi_{0}$ defined in Theorem 5.3.

Lemma 5.9. The product map $\phi_{0}$ defined in Theorem 5.3 factors through the evaluation map

$$
\mathrm{ev}_{*}: K_{*}\left(C_{\widetilde{B}, L, 0}^{*}(\widetilde{E})^{G}\right) \rightarrow K_{*}\left(C_{L, 0}^{*}(\widetilde{E})^{G}\right) .
$$

That is, for any $m, n \in\{0,1\}$ there exists a map

$$
\phi_{L, 0}: K_{m}\left(C_{L}^{*}(\widetilde{B})^{H}\right) \otimes K_{n}\left(C_{L, 0}^{*}(\widetilde{E}, \widetilde{B})^{G}\right) \longrightarrow K_{m+n}\left(C_{\widetilde{B}, L, 0}^{*}(\widetilde{E})^{G}\right)
$$

such that the following diagram commutes

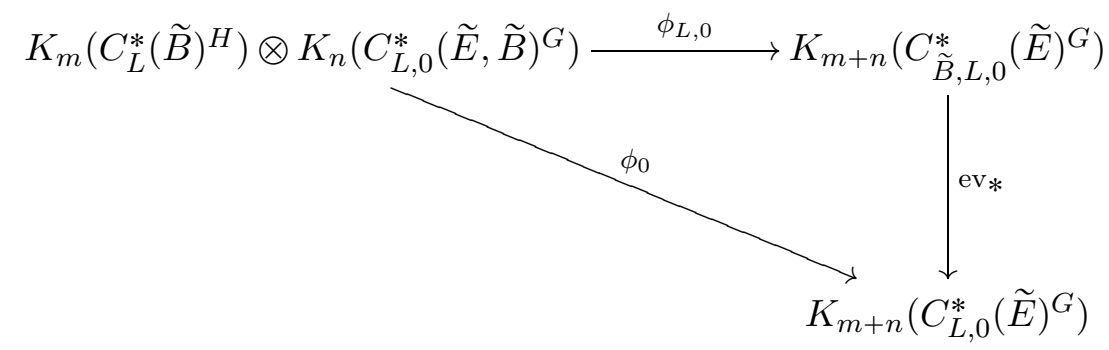


Proof. Without loss of generality, we assume that both $m$ and $n$ are zero. With the same notations as in the proof of Theorem 5.3, we define $\phi_{L, 0}$ by

$$
\phi\left(\left[f_{t}\right] \otimes\left[g_{t}\right]\right)=\left[\left(f_{t+s-1}-1\right) \otimes\left(g_{t}-1\right)+1\right],
$$

where $\left(f_{t+s-1}-1\right) \otimes\left(g_{t}-1\right)+1 \in\left(C_{L, 0}^{*}(\widetilde{E})^{G}\right)^{+}$is given by

$$
\begin{aligned}
& \left(\left(\left(f_{t+s-1}-1\right) \otimes\left(g_{t}-1\right)\right) h\right)(x, y) \\
= & \int_{\widetilde{B}} \int_{\widetilde{F}}\left(f_{t+s-1}-1\right)\left(x, x^{\prime}\right) \otimes\left(g_{t}-1\right)_{x^{\prime}}\left(y, y^{\prime}\right) h\left(x^{\prime}, y^{\prime}\right) d y^{\prime} d x^{\prime},
\end{aligned}
$$

with $h \in L^{2}(\widetilde{E})$. Here $t \in[1,+\infty)$ is the parameter in $C_{L, 0}^{*}(\widetilde{E})^{G}$ and $s \in[1,+\infty)$ is the extra parameter induced in $C_{\widetilde{B}, L, 0}^{*}(\widetilde{E})^{G}$. The expression makes sense as we may assume that the propagation of $f_{t}$ is small enough. After passing to the matrix algebra and the Grothendieck group, we obtain the map $\phi_{L, 0}$. The commuting diagram follows directly from the definition.

Lemma 5.10. With the same notations, for the fiberwise homotopy equivalence $f$, there exists a $K$-theory class $\rho_{L}(f) \in K_{\operatorname{dim} E}\left(C_{\widetilde{B}, L, 0}^{*}(\widetilde{E})^{G}\right)$ such that $\mathrm{ev}_{*}\left(\rho_{L}(f)\right)=\rho(f) \in K_{\operatorname{dim} E}\left(C_{L, 0}^{*}(\widetilde{E})^{G}\right)$.

Proof. Denote by $g^{B}, g^{E^{\prime}}$ and $g^{E}$ the metric on $B, E^{\prime}$ and $E$ respectively. For any $r \in[0,1]$ and $n \in \mathbb{N}^{+}$, let $\coprod_{n} B_{n+r}$ be the disjoint union of countably many $B$ 's, where $B_{n+r}$ is equipped with the metric $(n+r) g^{B}$. Similarly, we define $\coprod_{n} E_{n+r}^{\prime}$ and $\coprod_{n} E_{n+r}$, where $E_{n+r}^{\prime}$ and $E_{n+r}$ are equipped with the metric $g^{E^{\prime}}+(n+r) \pi^{* *} g^{B}$ and $g^{E}+(n+r) \pi^{*} g^{B}$.

Now we have the following fibrations

$$
F^{\prime} \longrightarrow \coprod_{n} E_{n+r}^{\prime} \stackrel{p^{\prime}}{\longrightarrow} \coprod_{n} B_{n+r} \text { and } F \longrightarrow \coprod_{n} E_{n+r} \stackrel{p}{\longrightarrow} \coprod_{n} B_{n+r},
$$

and the fiberwise homotopy equivalence $\coprod_{n} f_{n+r}=\coprod f$

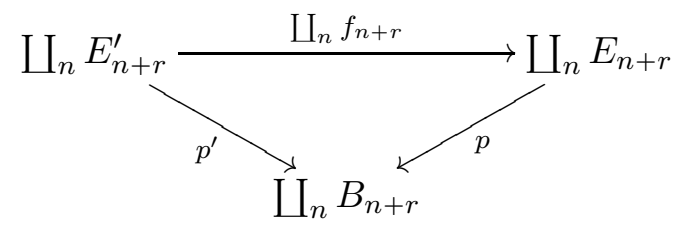

Since $f$ preserve the fibration, it induces a family higher rho invariant as in Definition 3.5

$$
\rho\left(\coprod_{n} f_{n+r}\right) \in K_{\operatorname{dim} E}\left(C_{L, 0}^{*}\left(\coprod_{n} \widetilde{E}_{n+r}\right)^{G}\right) .
$$


Hence for some $\varepsilon>0$ small enough, $\rho\left(\coprod_{n} f_{n+r}\right)$ admits a $K_{0}\left(\right.$ resp. $\left.K_{1}\right)$ representative which is a $\varepsilon$-almost projection (resp. unitary) with finite propagation uniformly in $n \in \mathbb{N}^{+}$. Along $\widetilde{B}$ the propagation of such representative restricted to $\widetilde{E}_{n+r}$ goes to zero as $n$ goes to infinity.

When $r$ varies in $[0,1]$, the above construction gives rise to a path $\rho_{L}(f)(s)$ for $s \in[1,+\infty)$, along which the propagation along $\widetilde{B}$ goes to zero as $s$ goes to infinity. Therefore the path defines a class in $K_{\operatorname{dim} E}\left(C_{\widetilde{B}, L, 0}^{*}(\widetilde{E})^{G}\right)$, which we will also denote by $\rho_{L}(f)(s)$. Furthermore, $\rho_{L}(f)(1)$ represents the same $K$-theory class as $\rho(f)$ in $K_{\operatorname{dim} E}\left(C_{L, 0}^{*}(\widetilde{E})^{G}\right)$ by definition. This finishes the proof.

Now we are ready to prove Theorem 5.5. We will go through the proof in details only for the case where the dimension of $B$ and $F$ are both even. The other cases are totally similar.

Proof of Theorem 5.5. Let $\rho_{L}(f)$ be as constructed in the proof of Lemma 5.10. We shall show that

$$
\phi_{L, 0}\left(\left[D_{B}^{s g n}\right] \otimes \rho(f ; B)\right)=\rho_{L}(f) \in K_{0}\left(C_{\widetilde{B}, L, 0}^{*}(\widetilde{E})^{G}\right)
$$

by the Mayer-Vietoris arguments. And the theorem follows from Lemma 5.9 and 5.10 .

We first assume a special case where $E=F \times B$, a trivial fiber bundle over $B$. In this case, the family algebra $C_{L, 0}^{*}(\widetilde{E}, \widetilde{B})^{G}$ is isomorphic to $C(B) \otimes C_{L, 0}^{*}(\widetilde{F})^{\Gamma}$. The product map $\phi_{L, 0}$ and the localized higher rho invariant $\rho_{L}(f)$ are constructed in Lemma 5.9 and 5.10 respectively. Using the same construction as in Section 4, we will obtain line (5.3) for this trivial case.

Now we turn to the general situation. For simplicity, we assume that the base space $B$ admits a triangulation that makes it a simplicial complex. Assume that the diameter of every simplex on $B$ is small enough so that the restriction of $E$ on every simplex is trivial. Let $B^{(k)}$ be a small open neighborhood of the $k$-skeleton of $B$, which contain the $k$-skeleton of $B$ as a deformation retraction. In particular, $B^{(k)}=B$ when $k$ is $\operatorname{dim} B$. Denote the lift of $B^{(k)}$ to $\widetilde{B}$ by $\widetilde{B}^{(k)}$ and the restriction of $\widetilde{E}$ to $\widetilde{B}^{(k)}$ by $\widetilde{E}_{\widetilde{B}^{(k)}}$.

For any $K$-theory element in $K_{*}\left(C_{\widetilde{B}, L, 0}^{*}(\widetilde{E})^{G}\right)$, its restriction to $\widetilde{E}_{\widetilde{B}^{(k)}}$ is well defined by multiplying the element by the characteristic function of $\widetilde{E}_{\widetilde{B}^{(k)}}$ on both side. Similarly for $K_{*}\left(C_{L}^{*}(\widetilde{B})^{H}\right)$ and $K_{*}\left(C_{L}^{*}(\widetilde{E}, \widetilde{B})^{G}\right)$. We will prove that line (5.3) holds when restricted to $\widetilde{E}_{\widetilde{B}^{(k)}}$ by induction on $k$.

When $k$ is zero, $B^{(0)}$ is a disjoint union of small balls in $B$, to which the restriction of $E$ is trivial. Therefore line (5.3) holds on $\widetilde{E}_{\widetilde{B}^{(0)}}$. Now we assume 
that line (5.3) holds on $\widetilde{E}_{\widetilde{B}^{(k)}}$. Let $\Delta$ be the disjoint union of the interior of every $k+1$-simplex in $B^{(k+1)}$. Denote the lift of $\Delta$ to $\widetilde{B}$ by $\widetilde{\Delta}$ and the restriction of $\widetilde{E}$ to $\widetilde{\Delta}$ by $\widetilde{E}_{\widetilde{\Delta}}$. Note that $B^{(k+1)}=\Delta \cup B^{(k)}$. By Proposition 5.8 , we have the following six-term exact sequence:

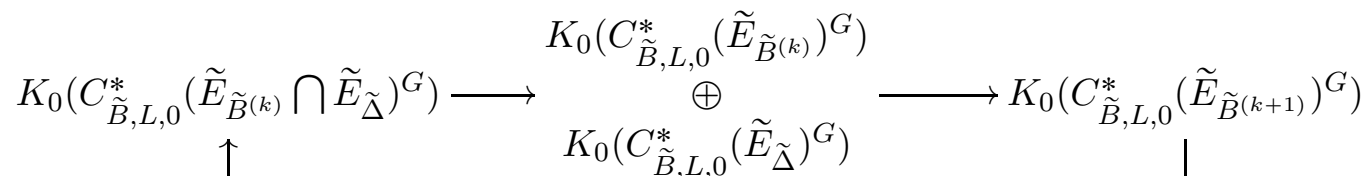

$$
\begin{aligned}
& K_{1}\left(C_{\widetilde{B}, L, 0}^{*}\left(\widetilde{E}_{\widetilde{B}^{(k+1)}}\right)^{G}\right) \longleftarrow K_{0}\left(C_{\widetilde{B}, L, 0}^{*}\left(\widetilde{E}_{\widetilde{\Delta}}\right)^{G}\right)
\end{aligned}
$$

From the assumption that the diameter of each simplex of $B$ is small, the restriction of $E$ to $\Delta$ or $\Delta \cap B^{(k)}$ is a disjoint union of trivial bundles. Direct computations show that

$$
\partial\left(\phi_{L, 0}\left(\left[D_{B}^{s g n}\right] \otimes \rho(f ; B)\right)-\rho_{L}(f)\right)
$$

is trivial in $K_{1}\left(C_{\widetilde{B}, L, 0}^{*}\left(\widetilde{E}_{\widetilde{B}^{(k)}} \cap \widetilde{E}_{\widetilde{\Delta}}\right)^{G}\right)$, thus it lies in the image of the map

$$
\begin{gathered}
K_{0}\left(C_{\widetilde{B}, L, 0}^{*}\left(\widetilde{E}_{\widetilde{B}^{(k)}}\right)^{G}\right) \\
K_{0}\left(C_{\widetilde{B}, L, 0}^{*}\left(\widetilde{E}_{\widetilde{\Delta}}\right)^{G}\right)
\end{gathered} \rightarrow K_{0}\left(C_{\widetilde{B}, L, 0}^{*}\left(\widetilde{E}_{\widetilde{B}^{(k+1)}}\right)^{G}\right)
$$

Then along with the inductive hypothesis, the $K$-theory classes represented by

$$
\phi_{L, 0}\left(\left[D_{B}^{s g n}\right] \otimes \rho(f ; B)\right)-\rho_{L}(f)
$$

restricted to $\widetilde{E}_{\widetilde{\Delta}}, \widetilde{E}_{\widetilde{B}^{(k)}}$ vanishes, which shows that

$$
\phi_{L, 0}\left(\left[D_{B}^{s g n}\right] \otimes \rho(f ; B)\right)-\rho_{L}(f)
$$

is the image of trivial class. Now line (5.3) follows when $k=\operatorname{dim} B$. 


\section{For special fiber bundle}

In this section, we show that Theorem 5.4 implies the product formula of numerical signature on fibered manifold given by Chern, Hirzebruch and Serre in $[1]$.

Consider the fiber bundle $\pi: E \rightarrow B$ with fiber $F$, with all those spaces are $4 k$-dimensional oriented closed Riemannian manifolds. Assume that $\pi_{1}(B)$ acts trivially on $H_{d R}^{*}(E)$, the de Rham cohomology of $E$. We would like to use our product formula to prove the original formula introduced by Chern, Hirzebruch and Serre in [1], namely

$$
\operatorname{sgn}(B) \times \operatorname{sgn}(F)=\operatorname{sgn}(E) .
$$

Consider the K-theoretic index map

$$
\operatorname{ind}_{E}: K_{0}\left(C_{L}^{*}(\tilde{E})^{\pi_{1}(E)}\right) \rightarrow K_{0}\left(C_{L}^{*}(p t)\right) \cong \mathbb{Z}
$$

induced by the map that crashes the whole space to a point and forgets the group action. Under this the localized index of signature operator will be mapped to its graded Fredholm index, i.e. $\operatorname{sgn}(E)$. Besides we replace $E$ by the base space $B$ and obtain

$$
\operatorname{ind}_{B}: K_{0}\left(C_{L}^{*}(\tilde{B})^{\pi_{1}(B)}\right) \rightarrow K_{0}\left(C_{L}^{*}(p t)\right) \cong \mathbb{Z} .
$$

Recall the equivariant family localization algebra $C_{L}^{*}(\tilde{E}, \tilde{B})^{\pi_{1}(E)}$ is the collection of some sections of a $\mathrm{C}^{*}$-bundle over $B$. Any element $s(t) \in C_{L}^{*}(\tilde{E}, \tilde{B})^{\pi_{1}(E)}$ is viewed as a family of operators $s(t)_{x} \in C_{L}^{*}(\tilde{F})^{\Gamma}$ for $x \in \tilde{B}$. Thus we define a family index map

$$
\left.\operatorname{ind}_{E, B}: K_{0}\left(C_{L}^{*}(\tilde{E}, \tilde{B})^{\pi_{1}(E)}\right) \rightarrow K_{0}(C(\tilde{B}))^{\pi_{1}(B)}\right) \cong K_{0}(C(B))
$$

by taking indices along the fiber.

Moreover, we have the following classical pairing of $K$-homology and $K$ theory

$$
\langle\cdot, \cdot\rangle: K_{0}\left(C_{L}^{*}(\tilde{B})^{\pi_{1}(B)}\right) \times K_{0}(C(B)) \rightarrow \mathbb{Z} .
$$

From the construction above, we see that the following diagram commutes.

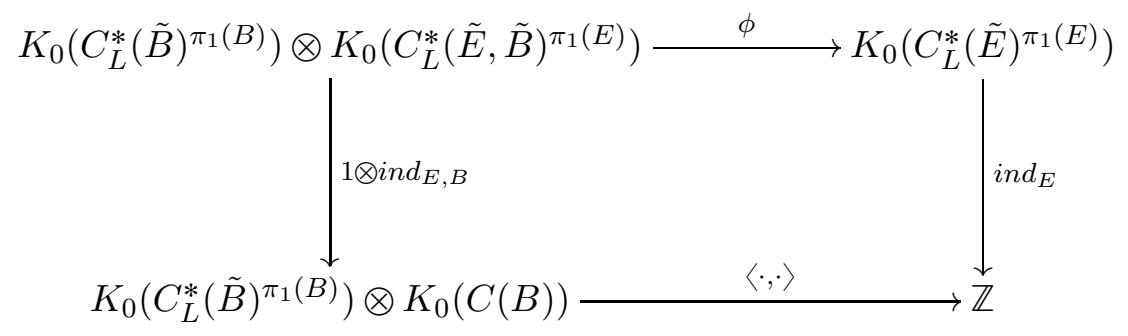


Therefore we have the identity

$$
\operatorname{sgn}(E)=\left\langle\left[D_{B}\right], \operatorname{ind}_{E, B}\left(\left[D_{E, B}\right]\right)\right\rangle .
$$

Since $F$ is even-dimensional, the family index $\operatorname{ind}_{E, B}\left(\left[D_{E, B}\right]\right)$ living in $K_{0}(C(B))$ can be viewed as a virtual vector bundle over $B$. The local picture of such vector bundle is $\left[k \operatorname{er}\left(D_{F}\right)\right]-\left[\operatorname{cok}\left(D_{F}\right)\right]$. As we have assumed that $\pi_{1}(B)$ acts on $H_{d R}^{*}(E)$ trivially, the virtual bundle is indeed a trivial bundle, i.e. it comes from the inclusion $\mathbb{Z} \cong K_{0}(C(p t)) \rightarrow K_{0}(C(B))$. Moreover, the preimage of $\operatorname{ind}_{E, B}\left(\left[D_{E, B}\right]\right)$ under the inclusion is actually

$$
\operatorname{dim} \operatorname{ker}\left(D_{F}\right)-\operatorname{dim} \operatorname{cok}\left(D_{F}\right)=\operatorname{sgn}(F) .
$$

Thus the pairing map $\langle\cdot, \cdot\rangle$ is simplified as followed

$$
\begin{aligned}
\left\langle\left[D_{B}\right], \operatorname{ind}_{E, B}\left(\left[D_{E, B}\right]\right)\right\rangle & =\left\langle\left[D_{B}\right], \operatorname{sgn}(F)\right\rangle \\
& =\operatorname{ind}_{B}\left(\left[D_{B}\right]\right) \times \operatorname{sgn}(F) \\
& =\operatorname{sgn}(B) \times \operatorname{sgn}(F) .
\end{aligned}
$$

From this we obtain the classical product formula of signature of Chern, Hirzebruch and Serre.

\section{References}

[1] S. S. Chern, F. Hirzebruch, and J.-P. Serre. On the index of a fibered manifold. Proc. Amer. Math. Soc., 8:587-596, 1957.

[2] N. Higson and J. Roe. Mapping surgery to analysis. I. Analytic signatures. K-Theory, 33:277-299, 2005.

[3] N. Higson and J. Roe. Mapping surgery to analysis. II. Geometric signatures. K-Theory, 33:301-324, 2005.

[4] N. Higson and J. Roe. Mapping surgery to analysis. III. Exact sequences. K-Theory, 33(4):325-346, 2005.

[5] N. Higson, J. Roe, and G. Yu. A coarse Mayer-Vietoris principle. Math. Proc. Cambridge Philos. Soc., 114(1):85-97, 1993.

[6] M. Hilsum and G. Skandalis. Invariance par homotopie de la signature à coefficients dans un fibré presque plat. J. Reine Angew. Math., 423:73-99, 1992.

[7] B. Jiang and H. Liu. Additivity of higher rho invariant for topological structure group in a differential point of view. arXiv:1804.09026, 2019. 
[8] P. Piazza and T. Schick. The surgery exact sequence, K-theory and the signature operator. Ann. K-Theory, 1(2):109-154, 2016.

[9] P. Siegel. Homological calculations with the analytic structure group. ProQuest LLC, Ann Arbor, MI, 2012. Thesis (Ph.D.)-The Pennsylvania State University.

[10] X. Tang, R. Willett, and Y.-J. Yao. Roe $C^{*}$-algebra for groupoids and generalized Lichnerowicz vanishing theorem for foliated manifolds. Math. Z., 290(3-4):1309-1338, 2018.

[11] C. Wahl. Product formula for Atiyah-Patodi-Singer index classes and higher signatures. J. K-Theory, 6(2):285-337, 2010.

[12] C. Wahl. Higher $\rho$-invariants and the surgery structure set. J. Topol., 6(1):154-192, 2013.

[13] S. Weinberger, Z. Xie, and G. Yu. Additivity of higher rho invariants and nonrigidity of topological manifolds. To appear in Communications on Pure and Applied Analysis.

[14] Z. Xie and G. Yu. Positive scalar curvature, higher rho invariants and localization algebras. Adv. Math., 262:823-866, 2014.

[15] Z. Xie and G. Yu. Higher invariants in noncommutative geometry. arXiv:1905.12632, To appear in the special volume dedicated to Alain Connes' 70th birthday, 2019.

[16] G. Yu. Localization algebras and the coarse Baum-Connes conjecture. K-Theory, 11(4):307-318, 1997.

[17] R. Zeidler. Positive scalar curvature and product formulas for secondary index invariants. J. Topol., 9(3):687-724, 2016.

[18] V. F. Zenobi. Mapping the surgery exact sequence for topological manifolds to analysis. J. Topol. Anal., 9(2):329-361, 2017. 